\title{
Scale Dependence of Atmosphere-Surface Coupling Through Similarity Theory
}

\section{Cedrick Ansorge ${ }^{1}$ (D)}

Received: 1 February 2018 / Accepted: 9 August 2018 / Published online: 25 August 2018

(c) The Author(s) 2018

\begin{abstract}
Monin-Obukhov similarity theory (MOST) applies for homogeneous and stationary conditions but is used in ever more complex and heterogeneous configurations. Here, it is used to estimate the surface friction velocity $u_{\star}$ from the wind speed and temperature in the atmospheric surface layer (ASL). Filters of varying scale and direction are applied to wind speed and temperature in the ASL before MOST is used to estimate $u_{\star}$. This procedure unveils the scale dependence of coupling between the ASL and the surface. Direct numerical simulation of turbulent Ekman flow above a smooth surface is used to explicitly resolve the near-wall dynamics. It is found that the viscous sub-layer may cease to exist, even in continuously turbulent neutral conditions, while the ASL covers more than one decade of variation in height. An underestimation in the variance of $u_{\star}$ estimated through MOST versus its actual variance is quantified as a function of height, averaging time, and length scale. For large filter scales, the variance exhibits a purely statistical convergence-there is no signature of long-term memory beyond the scale of coherent turbulent motion. Joint convergence of $u_{\star}$ estimated by MOST and the actual $u_{\star}$ is obtained for filter scales beyond several thousand wall units, and only for data filtered along both horizontal dimensions; the three-dimensional structure of the turbulence elements limits the convergence of data filtered along any of the single dimensions: time, the streamwise or spanwise direction. In stably stratified conditions, MOST is found to have no or negative skill in locally estimating ASL properties from $u_{\star}$ and should therefore only be applied to filtered quantities.
\end{abstract}

Keywords Boundary-layer turbulence · Flux-gradient relationship · Monin-Obukhov similarity theory $\cdot$ Taylor hypothesis

\section{Introduction}

The estimation of fluxes near the surface is a crucial task in numerical modelling of atmospheric flow and eminently linked to the yet unsolved problem of turbulence closure in classical physics. To close the flux-gradient problem at the surface, Monin-Obukhov similarity theory (MOST; Monin and Obukhov 1954) is commonly employed in numerical

Cedrick Ansorge

cedrick@posteo.de

1 Institut für Geophysik und Meteorologie, Universität zu Köln, Pohligstr. 3, 50969 Cologne, Germany 
formulations of the coupled land-atmosphere system. Scale similarity arguments and the analogy of the planetary boundary layer (PBL) with channel flow are exploited (i.e. outerlayer effects and the rotation of the reference frame are neglected; Sect. 2.1) to estimate the fluxes of heat and momentum at the surface. Motivated by tower measurements, MOST is conceptually designed in an ensemble-averaged context. Non-local effects due to advection corresponding to the formation of a (sequence of) internal boundary layers and corresponding jumps in the vertical profile (Garratt 1990) are neglected as well as the aggregation of fluxes, which further implies the limit of an infinite homogeneous surface. This limit is, however, rarely encountered in the coupled land-atmosphere system that is characterized by broadscale heterogeneities in virtually all quantities (Adrian 2007; Jiménez 2012; Simmer et al. 2015; Vereecken et al. 2016). Accounting for these heterogeneities and associated processes and interactions, both the atmospheric and surface components of coupled land-atmosphere systems have evolved at an increasing pace over the past few decades in terms of complexity and increasing resolution. However, MOST is still the essential methodology communicating perturbations, and not only the mean state, in between the land and atmospheric components. Despite these known conceptual and practical deficiencies, both in general (Kawai and Larsson 2012; Park and Moin 2014) and over heterogeneous surfaces in particular (Shao et al. 2013), MOST is commonly used to estimate surface fluxes under real-world conditions in virtually all global circulation models and also large-eddy simulation (LES) of atmospheric boundary-layer flow where wall models are commonly used.

Since its introduction, the validity of MOST under strong stable stratification, and in particular its limit of validity, has been a matter of debate. Modifications of similarity theory have been suggested and proven successful under certain conditions which take into account non-locality or local absence of turbulence (Nieuwstadt 1984; Gryning et al. 2007; Zilitinkevich and Esau 2007; Grachev et al. 2012; Optis et al. 2014). Further modifications include the effect of a partial-slip surface such as (wavy) water (Charnock 1955; Fairall et al. 2003; $\mathrm{Li}$ et al. 2018). For heterogeneous surfaces, the mosaic/tile approach is commonly employed (Avissar and Pielke 1989; Claussen 1991; Giorgi and Avissar 1997; Ament and Simmer 2006) which assumes validity of similarity theory over individual patches at the subgrid scale but takes into account the aggregation of fluxes at the scale of the grid. Far less attention has been devoted to another violation of the theoretical assumptions, namely the local variation of wall-shear stress $u_{\star}$; even though $u_{\star}$ is known to vary by about $40 \%$ around its mean (Alfredsson et al. 1988), a systematic assessment of the scale margins has, to the author's knowledge, remained elusive.

Assessment of the validity of MOST eliminates the possibility to use models where it is employed implicitly or explicitly, including standard LES approaches for atmospheric flows which use MOST as a wall model to link the surface to the first atmospheric level. We resort here to the direct numerical simulation (DNS) of a turbulent Ekman flow above a smooth surface as a physical model of the PBL. Still, the numerical simulation cannot achieve the Reynolds numbers commonly encountered in geophysical boundary layers (Moin and Mahesh 1998); recently, however, data at a sufficiently high Reynolds number and domain size to allow for a well-resolved logarithmic part of the wind speed profile have become available (Ansorge and Mellado 2016), and herein, an additional simulation with a further decrease of viscosity by about $70 \%$ is added. One reason why MOST is used so widely is its applicability in different stratification regimes; here, we limit the scope to stable and neutral stratification.

This work inquires as to what period of time and/or what area of space is appropriate to apply MOST locally to within a sufficient accuracy? And, what does this imply for the validity margins of similarity theory in terms of temporal and spatial scales? This is done with 
due regard of the high variability within the viscous sub-layer that acts as a lower boundary condition for the ASL (Sect. 3). The investigation of scale limits in both neutral (Sect. 4) and stable conditions (Sect. 5) provides, based on first principles, a spatial and temporal scale above which the interaction between the land surface and the boundary layer can be modelled by MOST given a desired representation of the local variability.

Such a systematic convergence quantification for one-point statistics of first and second order has implications for both numerical and observational studies of the PBL. It provides limits and biases for the estimation of mean profiles and fluxes from purely local samples, such as towers, or from limited-space observations such as lidar scans which are increasingly being used to investigate the PBL. These errors also propagate into the turbulence models calibrated based on such biased measurements. The impact of unveiling and quantifying these convergence issues, however, goes far beyond the implications for boundary-layer meteorology. It is closely related to the validity of Taylor's hypothesis (cf. Sect 4.2), an intensely debated issue in fluid dynamics over the past decades (Lin 1953; Wills 1964; Krogstad et al. 1998; Moin 2009).

\section{Simulation and Analysis Methods}

\subsection{Formulation of Monin-Obukhov Similarity Theory}

Monin-Obukhov Similarity theory was introduced to the international research community by Monin (1970) and is accepted as a valid model for the estimation of surface fluxes since the great success of the Kansas (Haugen et al. 1971) and Minnesota (Haugen et al. 1976) experiments and a revaluation of the underlying universal functions by Högström (1988, 1996). The impact of stratification is considered via a supposedly universal non-dimensional stability correction function based on the non-dimensional height $\zeta \equiv z / L$ (Obukhov 1971; Businger and Yaglom 1971) (translated from Obukhov 1946). Here, the Obukhov length $L$ is defined by

$$
L \equiv \frac{u_{\star}^{2}}{b_{\star}}
$$

with

$$
u_{\star}^{2} \equiv \sqrt{\tau_{x z}^{2}+\tau_{y z}^{2}}=v \sqrt{\left(\left.\partial_{z} u\right|_{z=0}\right)^{2}+\left(\left.\partial_{z} v\right|_{z=0}\right)^{2}},
$$

and

$$
\left.u_{\star} b_{\star} \equiv \frac{v}{\operatorname{Pr}} \frac{\partial b}{\partial z}\right|_{z=0},
$$

where $v$ denotes kinematic viscosity of the fluid, $P r$ is the Prandtl number and we let $P r=1$, very close to the actual value of $P r \approx 0.7$ for air in typical atmospheric conditions. Buoyancy is denoted by $b$ and can be interpreted commensurate with potential temperature as the Boussinesq approximation is used. The friction velocity and buoyancy are denoted by $u_{\star}$ and $b_{\star}$. The no penetration boundary condition $\left.w\right|_{z=0}=0$ is employed in Eq. $1 \mathrm{~b}$ to simplify the wall-stress tensors $\tau_{x z}$ and $\tau_{y z}$. Note that viscous fluxes at the wall are used here in defining the Obukhov length, a consequence of eliminating the subgrid-scale closure together with an aerodynamically smooth wall. Sometimes, the non-dimensional von Kármán constant $\kappa$ is 
included in the definition of $L$. We refrain here from this practice as it introduces an additional parameter to the problem of stability dependence. ${ }^{1}$

The non-dimensional gradient of wind speed in the stratified surface layer $\Phi_{M}$ is

$$
\left(\frac{\kappa z}{u_{\star}}\right) \frac{\partial U}{\partial z}=\Phi_{M}
$$

where the instantaneous velocity components are denoted by $u, v$ and $w$ in the streamwise, spanwise and vertical direction, and uppercase letters $U, V$ and $W$ refer to the corresponding domain averages. The geometric height above the aerodynamically smooth surface is denoted by $z$. An uppercase ' + ' denotes normalization by viscous wall units $\left(u_{\star}, v\right)$ and an uppercase '-' normalization by outer scales $(G, f)$,

$$
\begin{aligned}
& z^{+}=z u_{\star} / v \\
& z^{-}=z / \delta,
\end{aligned}
$$

where $\delta=u_{\star} / f$ is the boundary-layer depth scale of the turbulent flow. Correspondingly, $u^{+}=u / u_{\star}, b^{+}=b / b_{\star}$ and $t^{-}=t f /(2 \pi)$.

Assuming a linear stability correction function $\Phi_{i}(\zeta) \equiv 1+\beta_{i} \zeta$ (with $i=M, H$ representing momentum and buoyancy profiles respectively) the Businger-Dyer relationship is obtained:

$$
\begin{aligned}
\int_{z_{0}}^{z} \mathrm{~d} U & =\int_{z_{0}}^{z} u_{\star} \frac{\Phi_{M}}{\kappa z^{\prime}} \mathrm{d} z^{\prime} \Rightarrow U(z)=\frac{u_{\star}}{\kappa}\left[\ln \left(\frac{z}{z_{0}}\right)+\frac{\beta_{M}\left(z-z_{0}\right)}{L}\right]+U\left(z_{0}\right), \\
\int_{z_{0}}^{z} \mathrm{~d} B & =\int_{z_{0}}^{z} b_{\star} \frac{\Phi_{H}}{\kappa z^{\prime}} \mathrm{d} z^{\prime} \Rightarrow B(z)=\frac{b_{\star}}{\kappa}\left[\ln \left(\frac{z}{z_{0}}\right)+\frac{\beta_{H}\left(z-z_{0}\right)}{L}\right]+B\left(z_{0}\right) .
\end{aligned}
$$

The term $\beta_{i} z_{0} / L$ is the stability correction at $z=z_{0}$ and with $z_{0} \ll L$ one may assume $\Phi_{M}\left(z=z_{0}\right)=\Phi_{H}\left(z=z_{0}\right)=1$. In addition,

$$
\beta_{M} \zeta=\Phi_{M}-1=\kappa\left[\frac{U(z)}{u_{\star}}-\frac{\ln \left[z / z_{0}\right]}{\kappa}\right]+\kappa\left[\frac{U\left(z_{0}\right)}{u_{\star}}-\frac{\beta_{M} z_{0}}{L}\right] .
$$

The stability correction can hence be estimated from the difference between the neutral and stratified profiles at a fixed geometric height $z$ by

$$
\begin{aligned}
& \beta_{M} \zeta \approx \kappa\left[U^{+}(z)-U_{\mathrm{N}}^{+}(z)\right]-\kappa\left[U^{+}\left(z_{0}\right)-U_{\mathrm{N}}^{+}\left(z_{0}\right)\right] \\
& \beta_{M} \zeta \approx \kappa \Delta U^{+}(z)
\end{aligned}
$$

where $U_{\mathrm{N}}^{+}$refers to the profile of the streamwise velocity component under truly neutral conditions, i.e. for $R i_{B}=0$, and $\Delta U^{+}(z)$ is the difference between the case under consideration and $U_{\mathrm{N}}^{+}(z)$. Further, the approximation $\Delta U^{+}\left(z_{0}\right) \approx 0$ is used, which is justified when $z_{0} \ll L$ and $z_{0} \ll z$ for any $z$ within the ASL.

\subsection{Set-Up and Numerical Method}

The set-up here is similar to that by Coleman et al. (1990) (cf. Ansorge and Mellado 2014): governing flow equations are solved under the Boussinesq approximation in a horizontally

1 Due to omission of $\kappa$ in the definition of $L$, the non-dimensional height $\zeta$ differs from that introduced by Obukhov (1946) by a factor $\kappa$. For comparison, let $L_{\kappa}=u_{\star}^{2} /\left(\kappa b_{\star}\right)$ the classic Obukhov length including the von Kármán constant, and correspondingly $\zeta_{\kappa}=z / L_{\kappa}=z \kappa / L=\zeta \kappa$, which is important when comparing stability regimes. 


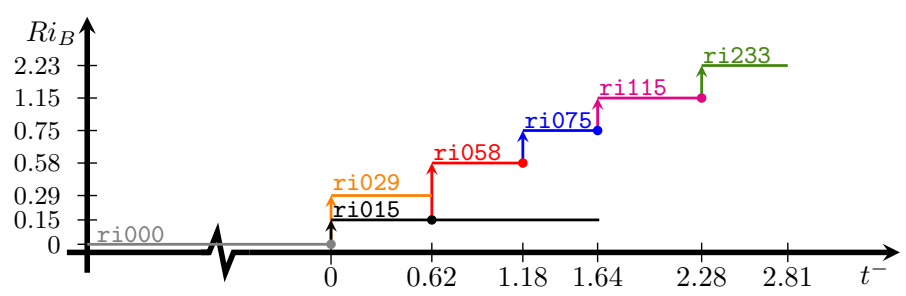

Fig. 1 Sketch showing schematically the sequence of simulations with increasing stratification as outlined in Table 1. Each line corresponds to one simulation where a dot illustrates the point in time at which the state of the simulation is used to initialize a case with higher stratification as indicated by the vertical arrows

doubly periodic flow domain. The boundary conditions on the velocities correspond to Ekman flow over a smooth wall. For the buoyancy, a Dirichlet-type boundary condition is used at the top and bottom of the domain, that is, the buoyancy difference between the wall and the free stream is fixed within each simulation. The parameters of this set-up are the geostrophic wind $\mathbf{G}$, the fluid kinematic viscosity $v$, the Coriolis parameter $f$ and the buoyancy difference $B_{0}$ between the wall and free stream. Let $G \equiv|\mathbf{G}|$ the geostrophic wind speed, and align the coordinate direction $O x$ with the surface friction (Sect. 2.3). The two dimensionless groups

$$
R e \equiv G \delta_{N} / v
$$

and

$$
R i_{B} \equiv B_{0} \delta_{\mathrm{N}} / G^{2}
$$

viz. the Reynolds and Richardson numbers, govern the flow evolution in time. Here, $\delta_{N}=$ $u_{\star N} / f$ is a measure of the boundary-layer height under neutral conditions. Note that this sets the eddy-turnover time $1 / f$ independent of $R e$ to leading order (the geostrophic drag coefficient $u_{\star} / G$ is only weakly dependent on $R e$, Spalart 1989) and as a fixed fraction of the inertial period - an inherent property of turbulent Ekman flow. In terms of $\delta_{95}$, the height at which the total stress is reduced to $5 \%$ of the wall shear stress, it is $\delta_{N} / \delta_{95} \approx 1.6$ under neutral stratification; under stable stratification, the PBL, however defined, is shallower. $R i_{B}$ is defined here in terms of $\delta_{\mathrm{N}}$ and thus characterizes the initial condition, which may be out of equilibrium, only. Thus, $R i_{B}$ has no immediate role in characterizing the bulk stratification of the PBL throughout the simulation (Ansorge and Mellado 2014).

Here, the governing parameters are varied to, (1) elucidate the impact of stratification, and (2) ascertain Reynolds-number independence to within a certain degree. The impact of stratification is assessed by a step-by-step increase in stratification $\left(R i_{B}\right)$ over time which creates a somewhat realistic buoyancy profile resulting from a turbulent simulation. Stratified simulations are labelled by $R i_{B}$ as $r i<\mathrm{XXX}>$ (cf. Table 1). The initial condition used for the cases ri014 and ri029 is a realization of the neutrally stratified case ri000, and the buoyancy profile is prescribed by an error function in the wall-normal direction. The stronger stratified cases ri058 and ri076 use intermediate states of cases ri014 respectively ri 058 as initial condition with the buoyancy field adjusted by multiplication to match the desired bulk stratification; the sequence of simulations over time with increasing stratification is schematically shown in Fig. 1. Regarding (2), simulations at $R e=2.6 \times 10^{4}$ and $R e=$ $0.77 \times 10^{4}$ (introduced by Ansorge and Mellado 2016) are complemented by a case at $R e=4.2 \times 10^{4}$; these cases are labelled by re_low, re_high and ri 000 as outlined in Table 1. 


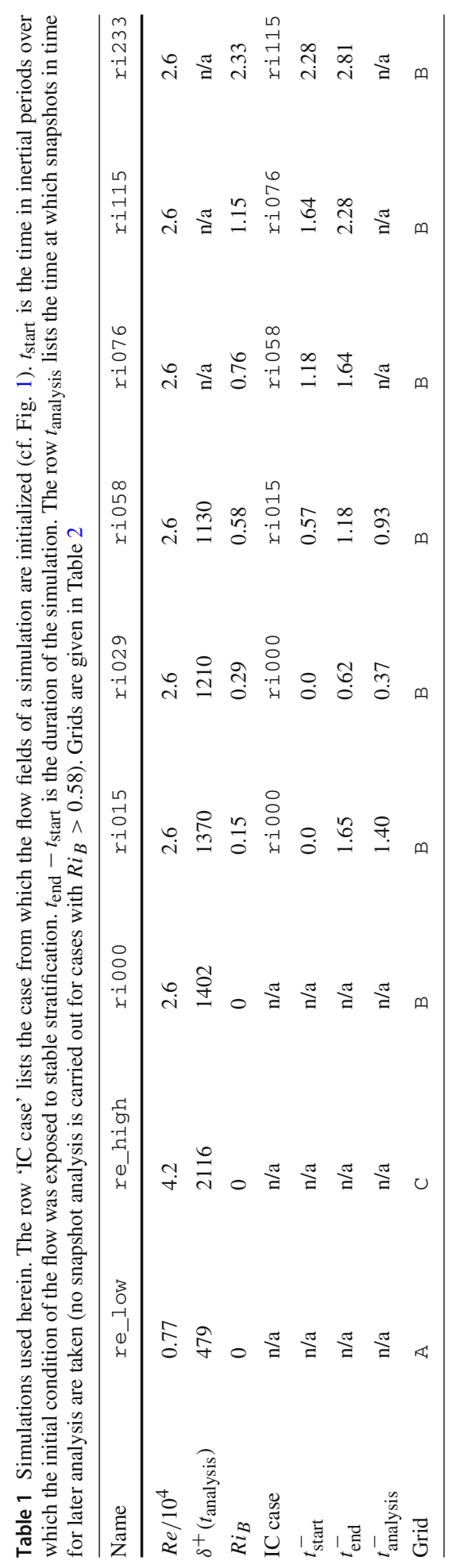


Table 2 Grid specifications. $N_{x, y, z}$ is the number of collocation points in the respective directions; $S_{x, y, z}$ denotes the box size as normalized by the Rossby radius $\Lambda \equiv G / f ; \Delta_{x, y, z}$ is the grid spacing. Extension of the domain in inner and outer units $\left(S_{x y}^{+}\right.$and $\left.S_{x y}^{-}\right)$is given here though not part of the domain specification

\begin{tabular}{lllllllll}
\hline Label & $N_{x}$ & $S_{x y} / \Lambda$ & $N_{y}$ & $N_{z}$ & $S_{z} / \Lambda$ & $\Delta x / \Lambda$ & $S_{x y}^{+}$ & $S_{x y}^{-}$ \\
\hline A & 2048 & 1.08 & 2048 & 192 & 0.17 & $5.27 \times 10^{-4}$ & $8.36 \times 10^{3}$ & 17.5 \\
B & 3072 & 1.08 & 6144 & 512 & 0.18 & $3.52 \times 10^{-4}$ & $2.86 \times 10^{4}$ & 20.4 \\
C & 2560 & 0.54 & 5120 & 640 & 0.18 & $2.11 \times 10^{-4}$ & $2.58 \times 10^{4}$ & 10.9 \\
\hline
\end{tabular}

\subsection{Frame of Reference}

Turbulent shear flow is commonly analyzed in a reference frame where one axis coincides with the direction of the shear. The rotation of wind direction with height in Ekman flow leaves several choices for the horizontal orientation of the reference frame. In particular, one may consider data aligned with the surface shear, with the direction of the height-local mean velocity and with the geostrophic velocity. Due to the pronounced anisotropy of surface streaks in the ASL, alignment of one grid component with the surface shear is a desired property, but can only be achieved a posteriori as the exact value of the surface veering is part of the (numerical) solution. For the numerical solution, the grid is rotated by about $20.5^{\circ}$ to approximately align the $O x$ component with the surface shear. In the framework of MOST, it is, however, crucial to analyze data consistently with the so-called channel-flow analogy, i.e. aligned with surface shear. To acquire exact alignment, all data are interpolated onto a grid that is rotated around the vertical axis to coincide with the direction of surface shear to within $\pm 0.1^{\circ}$.

\subsection{Analysis Method}

In order to assess the convergence of individual samples to their ensemble averages, both temporally-and spatially-resolved data are used. With regards to time-resolved data, the flow is sub-sampled at virtual towers, that is, in full temporal (every full Runge-Kutta iteration step) and vertical resolution (every vertical collocation point) but on a coarsened horizontal grid. This sampling approach is also advantageous with respect to observational atmospheric data. Such data are most often obtained from fixed-in-space towers at a high temporal and generally very coarse spatial resolution - if data are available within a spatial separation relevant to turbulent processes at all. In the set-up largest in terms of $\delta_{N}(R e=1000$, $S_{x y}^{-} \approx 20$ ), the towers are placed on a regular grid at $32 \times 32$ locations in each horizontal plane. This reduces the amount of information by about $2^{-10}$ with respect to the full grid and thus makes the amount of information tractable, also from a computational perspective. Separation of towers in physical space corresponds to a spacing of approximately $\left(0.64 \delta_{N} \times 0.64 \delta_{N}\right)$ or $\left(1.01 \delta_{95} \times 1.01 \delta_{95}\right)$. Taking $\delta_{95}$ as an estimator of the large-eddy size in this PBL, such a spatial separation allows one to consider each tower as exposed to an approximately independent realization of turbulence.

Let $\mathbf{x}_{i}(t, z)$ be an instantaneous flow realization at time $t$, height $z$ and location $\mathbf{r}_{i}$. Let further $\left(t_{n}\right)_{n \in \mathbb{N}}$ the discrete series of times through which the flow is integrated, and $\left(\mathbf{r}_{i}\right)_{i \in\{1 \ldots 1024\} \subset \mathbb{N}}$ the list of tower locations in the two-dimensional plane $O x y$. Further, define time-filtered instances of the local data as 
(a)

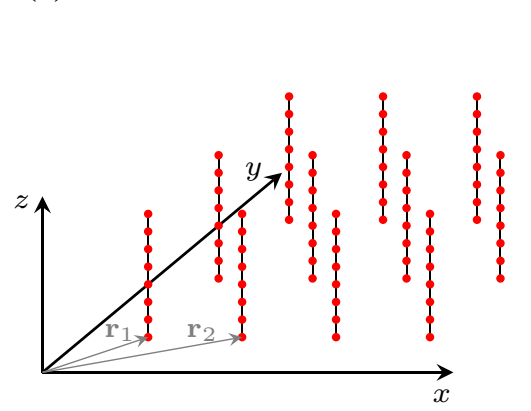

(b)

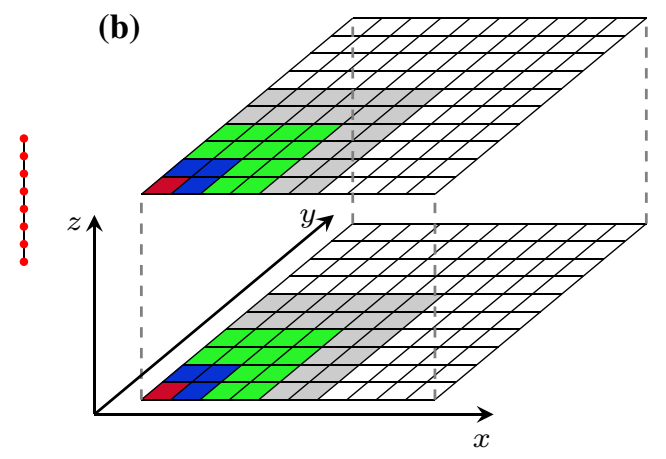

Fig. 2 Illustration of the tower probing approach employed in the convergence analysis for temporally resolved probes (left) and the horizontal coarse graining in $\mathcal{X} \mathcal{Y}$ employed in the convergence analysis for spatiallyresolved data (right)

$$
\mathcal{T}_{T}\left[x_{i}(z)\right]:=\frac{1}{n(T)} \sum_{n=1}^{n(T)} \mathbf{x}_{i}\left(t_{n}, z\right),
$$

with $n(T)$ such that $t_{n(T)-1}<T \leq t_{n(T)}$, that is, $\mathcal{T}_{T}\left[x_{i}(z)\right]$ is the average of the tower at $\mathbf{r}_{i}$ over the time period of length $T$. Ergodicity of the stationary, four-dimensional turbulent field implies

$$
\left\langle\mathbf{x}_{i}(t, z)\right\rangle=\lim _{T \rightarrow \infty} \mathcal{T}_{T}\left[x_{i}(z)\right],
$$

where angle brackets denote the expected value of an ensemble average, i.e.

$$
\langle x\rangle=\frac{1}{N} \sum_{j=1}^{N} x^{j},
$$

with $x$ any quantity and $x^{j}$ a specific realization of $x$, and herein commonly a flow sample.

For spatially-resolved data (available as sub-sampled in the time dimension), a corresponding approach is employed with the averaging over time in Eq. 5 replaced by an averaging over a successively increasing partition of space (as indicated in Fig. 2b). $\mathcal{X}_{S}, \mathcal{Y}_{S}$, and $\mathcal{X} \mathcal{Y}_{S}$ denote the horizontal spatial filter over the length scale $S$ operating on the spatially-resolved data along the streamwise $(O x)$, and spanwise $(O y)$ direction, and over a horizontal square $(O x y)$, respectively. This approach is sometimes referred to as coarse graining (Katsoulakis et al. 2008a, b; Aluie and Kurien 2011). Spatial coarse-graining of DNS data with the filter $\mathcal{X} \mathcal{Y}_{S}$ is equivalent to the filtering employed in the formulation of LES, suggesting a possible direct comparison of the data available here for a priori and a posteriori validation of LES subgrid closures and wall models also with respect to applications other than the scale dependence of surface-atmosphere exchange.

The scale dependence of the relation between wall friction and local wind speed in the ASL is investigated by analysis of such filtered data of local wall friction $\left(u_{\star \mathrm{F} l o c}^{\mathrm{F}}\right)$ and the wall friction estimated (modelled based on the ASL wind speed) through similarity theory $\left(u_{\star \text { mod }}^{\mathrm{F}}\right)$ :

$$
\begin{aligned}
& u_{\star}^{\mathrm{F}}=F\left[u_{\star l o c}\right], \\
& u_{\star \bmod }^{\mathrm{F}}=\tilde{u}_{\star}(F[u], F[b]),
\end{aligned}
$$


where $\tilde{u}_{\star}$ is the solution to the non-linear system of equations implied by the MOST equations with linearized stability correction (cf. Appendix 1 for details). $F$ is one of the filters $\mathcal{T}_{T}$ in time or $\mathcal{X}_{S}, \mathcal{Y}_{S}, \mathcal{X} \mathcal{Y}_{S}$ in horizontal space (Eq. 5). Due to the assumption of horizontal homogeneity in MOST, the estimates of $u_{\star \bmod }$ and $b_{\star \bmod }$ depend only on the filtered streamwise velocity component $F[U(z)]$, buoyancy $F[B(z)]$, and height $z$. Therefore, these estimates are free of self-correlation-a desirable property in the convergence analysis carried out henceforth.

The amount of data available allows consideration of the joint probability density functions (PDF) of $u_{\star}^{\mathrm{F}}$ and $u_{\star \text {, mod }}^{\mathrm{F}}$, which yields four parametric families of joint PDFs depending on the filtering applied to the data: $p d f^{\mathcal{T}_{T}}(z, t), p d f^{\mathcal{X}_{S}}(z, t), p d f^{\mathcal{Y}_{S}}(z, t)$, and $p d f^{\mathcal{X} \mathcal{Y}_{S}}(z, t)$. Each of these families depends on the height within the logarithmic layer where the wind speed and buoyancy fields are evaluated. The time dependence drops strictly in neutral conditions; in stable conditions, we only analyze data within the regime that the flow enters once the ASL is in quasi-equilibrium with the modified surface boundary condition. (Note that this may be the case despite the presence of an inertial oscillation which is very slow in comparison with ASL dynamics, even at the intermediate $R e$ considered here.)

\section{Friction Variability at the Surface and Thickness of the Viscous Sub-layer}

The logarithmic layer is often called the surface layer in geophysical applications, even if the associated scalings are only valid away from the surface. In fact, the $R e$-dependent threshold $z^{+}>3 \sqrt{\delta^{+}}$has been suggested as a lower bound for the surface-layer scaling of variances (Marusic et al. 2013) while $z^{+}>30$ appears to suffice in terms of the mean wind speed profiles (Sect.4). Considering the coupling between the surface and the ASL, the part of the flow in between the two is of crucial importance when the actual dynamics of the surfaceatmosphere exchange are examined. Hence, wall-modelled LES (or other methods using a wall model) cannot be used for our research goal as explicit resolution of the viscous sublayer and the buffer layer of the flow is essential. In DNS, the explicit resolution of near-wall dynamics eventually allows for the elimination of MOST from the set-up.

While the buffer layer around $10<z^{+}<20$ contains most of the turbulence production, the layer underneath, in immediate vicinity to the wall, is the viscous sub-layer. In the viscous sub-layer, velocity and scalar profiles approach their surface values with steep gradients, and viscous effects dominate. Hence, the bulk of momentum and heat is transported through viscous diffusion and the associated mean profiles of velocity and temperature become linear (Foken et al. 1978; Foken 2002), i.e.

$$
U^{+}\left(z^{+}\right)=z^{+}
$$

The thickness of the layer over which this relation holds for mean profiles is approximately five wall units for average profiles of a turbulent flow.

Variation of the local wall shear stress by almost one order of magnitude (Fig. 3) illustrates considerable variability of the flow-even within the viscous sub-layer where turbulent momentum exchange is small. We investigate here how variable the height of the viscous sub-layer is as a function of the local surface friction. As weak to moderate stratification is expected to have only a minor impact within the viscous sub-layer, we limit this investigation to neutral stratification. Therefore, the depth of the viscous sub-layer $\delta_{\text {visc }}$ is defined as the lowest height at which the non-dimensional streamwise velocity component deviates by more than $1 / 10$ from a linear profile, i.e. 

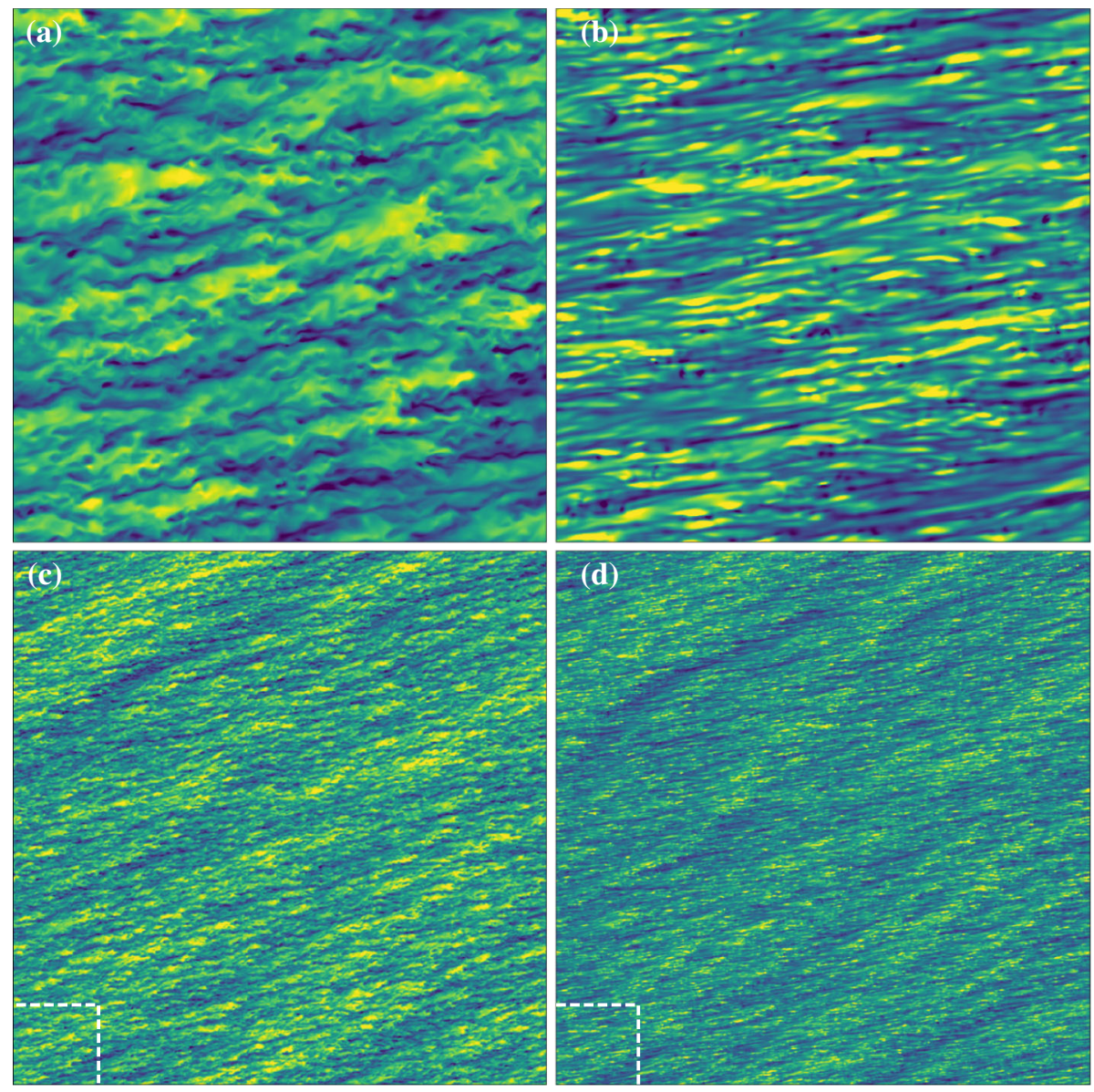

Fig. 3 Two-dimensional field of total horizontal wind speed $\sqrt{u^{2}+v^{2}} / G$ (left panels, $\mathbf{a}$ and $\mathbf{c}$, shown in the range $0.5<\left(\sqrt{u^{2}+v^{2}}\right) / G<1$ from blue to yellow) and wall friction velocity $u_{\star} / G$ (right panels, $\mathbf{b}$ and d, shown in the range $\left(0.03<u_{\star} / G<0.07\right.$ from blue to yellow $)$. Lower panel shows the full simulation domain $(2560 \times 5120$ grid points); Upper panel shows $1 / 8 \times 1 / 8$ of the domain as indicated by the dashed lines in the lower panel

$$
\delta_{\mathrm{visc}}=\min \left\{z:\left|u^{+\mathrm{loc}} / z^{+\mathrm{loc}}-1\right|>0.1\right\} .
$$

Here, a superscript + loc denotes local inner normalization, i.e. the instantaneous local surface shear stress $u_{\star l o c}$ is used in the wall scaling. It is noted that an approximately linear velocity profile up to a particular level does not suffice as proof of absence of turbulent stress; the rare continuation of the linear profile through the buffer layer may also occur by coincident appearance of a turbulent vortex from time to time. On the contrary, the deviation of the local profile from a linear one always indicates turbulent stress.

Most commonly, the thickness of the viscous sub-layer, $\delta_{\text {visc }}^{+}$, is in the range $2<z^{+}<6$, but excursions in the range $1<z^{+}<20$ occur (Fig. 4). A least-squares fit of all data points yields

$$
\left\langle\delta_{\mathrm{visc}}\right\rangle^{+} \approx 5.0+10 \frac{u_{\star \mathrm{loc}}}{G},
$$




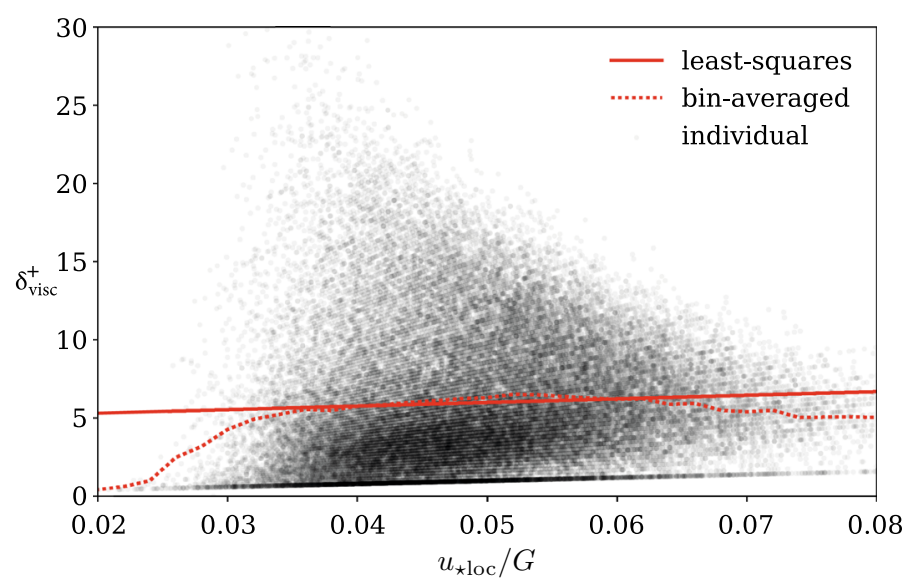

Fig. 4 Thickness of the viscous sub-layer as defined in Eq. 9 in global inner units $z^{+}$versus the non-dimensional local shear stress $u_{\star}$ loc $/ G$. The solid red line shows a least squares fit, and the dotted red line shows bin-averaged $\delta_{\text {visc }}$

which agrees well with the expected thickness of approximately five wall units and appears almost insensitive to the local friction velocity. (Note that $u_{\star} / G$, the surface friction normalized by the geostrophic wind speed, rarely acquires values larger than 0.1 (Fig. 4).) While the least-squares fit shows a slight increase of $\delta_{\text {visc }}^{+}$with the local friction $u_{\star}$ loc, for both high and low local friction the bin-averaged $\delta_{\text {visc }}^{+}$is substantially smaller. The minimum value of $\delta_{\text {visc }}$ according to Eq. 9 is $z_{1}$, the first grid point above surface, and it is $z_{1}^{+ \text {loc }}=z_{1} u_{\star l o c} / \nu$. This lowest possible value appears for all $u_{\star l \text { loc }} / G$, i.e. regardless of the local friction velocity, the viscous sub-layer may cease to exist. And this cessation is rather common, even in neutral conditions.

\section{Convergence to MOST in Neutral Conditions}

Much of the debate on the validity of, and problems associated with, MOST focuses on the impact of stratification and modifications of the stability correction functions or restrictions of the stability range over which the theory may be applied (Sect. 1). On the other hand, an important and theoretically relevant limit is neutral stratification: even though this limit is rarely encountered in atmospheric conditions, it is comparatively easily accessible through analytical and numerical investigation. Most importantly, neutral stratification corresponds to $\zeta=z / L=0$ and assumptions about the flow-dependence on $L$ are not required; the flux-profile relationship is then a fit to the well-known logarithmic wind-speed profile in the context of ensemble averages. In neutrally stratified Ekman flow, an equilibrium is acquired between the suppression of turbulence due to rotation and the boundary-layer growth over a non-rotating flat plate manifest in a statistically steady state. While there is debate on the universality of the von Kármán constant (Spalart et al. 2008), the extent of the logarithmic layer, and effects of entrainment (Ansorge and Mellado 2016), the existence of a layer over which the wind-speed profile adheres to an approximately logarithmic profile is a wellestablished consensus. Indeed, when considering the temporal and spatial average of the wind speed-profiles - the best estimate of the ensemble average available-our simulations exhibit a well-established logarithmic region that deepens as the Reynolds number increases (Fig. 5a). 

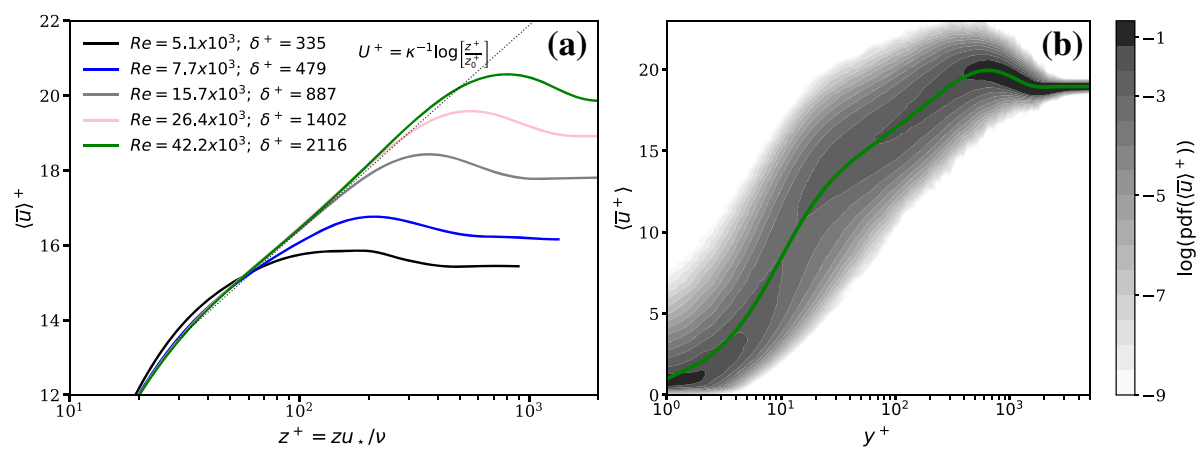

Fig. 5 a Mean profiles of the streamwise velocity component $U$ for simulations with Reynolds numbers varying from $R e=0.5 \times 10^{4}$ to $R e=4.2 \times 10^{4}$ (parameters of the logarithmic law for the mean wind speed are $\kappa=0.42$ and $\left.z_{0}^{+}=0.1\right)$. b Logarithm of the PDF of the velocity aligned with the surface shear stress for $R e=4.2 \times 10^{4}$ (case re_high); the thick green line shows the mean wind speed profile as in panel a

With turbulence being the defining flow property inside the logarithmic layer of a wallbounded flow, instantaneous profiles of $u$ are characterized by enormous variability (Fig. 5b). When applying MOST to estimate fluxes at the surface based on gradients, one postulates the covariation of wind speed in the immediate vicinity to the surface and higher up in the ASL. The general applicability of a logarithmic velocity profile is indeed a manifestation of this covariation in the mean profiles. It is, however, unclear to what extent the covariation of $u_{\star}$ and the local wind speed at a particular height within the ASL applies to instantaneous and filtered variations of the surface stress.

In fact, the viscous and buffer layers in between the surface and the logarithmic layer can act to propagate, damp, or even dynamically amplify a perturbation. When phrased in terms of the presentation in Fig. 5b, the question arises of if (and if so, how?) local deviations from the mean in the viscous sub-layer $\left(y^{+} \lesssim 5\right)$ are related to those in the ASL.

\subsection{Convergence and Scale Dependence in the Streamwise Direction and Time}

Consider now the validity of MOST for a neutrally-stratified Ekman flow when moving from the bulk limit of the ensemble average (represented here by time- and slab-averaged data) to the instantaneous limit of local-in-time or local-in-space data. Motivated by the common sampling of atmospheric towers in time and more recent methods which allow sampling along one-dimensional flow transects (Sayde et al. 2015), this section only investigates the one-dimensional convergence in space and time; we turn to the convergence in horizontal planes that is more relevant to numerical simulations of filtered equations of motions such as LES or RANS, in Sect. 4.3. For illustrative purposes, consider the two-dimensional fields of total horizontal wind speed $u_{H} \equiv \sqrt{u^{2}+v^{2}}$ and the local surface friction $u_{\star \text { loc }}$ (Fig. 3). Large-scale features appear strongly related (lower row) while there exist different smallscale structures in these fields (upper row), that suggests a scale dependence of the relation between ASL wind speed and surface friction.

To actually quantify the scale dependence, the joint PDFs of the filtered wall friction $u_{\star \text { loc }}^{\mathrm{F}}$ and estimated wall friction $u_{\star \text { mod }}^{\mathrm{F}}$ (Sect. 2.4) are presented in Fig. 6, where $F$ is the filter ${\stackrel{\mathcal{T}}{t_{\text {filt }}}}^{+}$ or $\mathcal{X}_{x_{\text {filt }}^{+}}$respectively. We resort here to the level $z^{+} \approx 32$ ( $z^{-} \approx 0.013$; case re_high), i.e. the lower end of the logarithmic region. If MOST was to hold exactly at all scales of motion, 

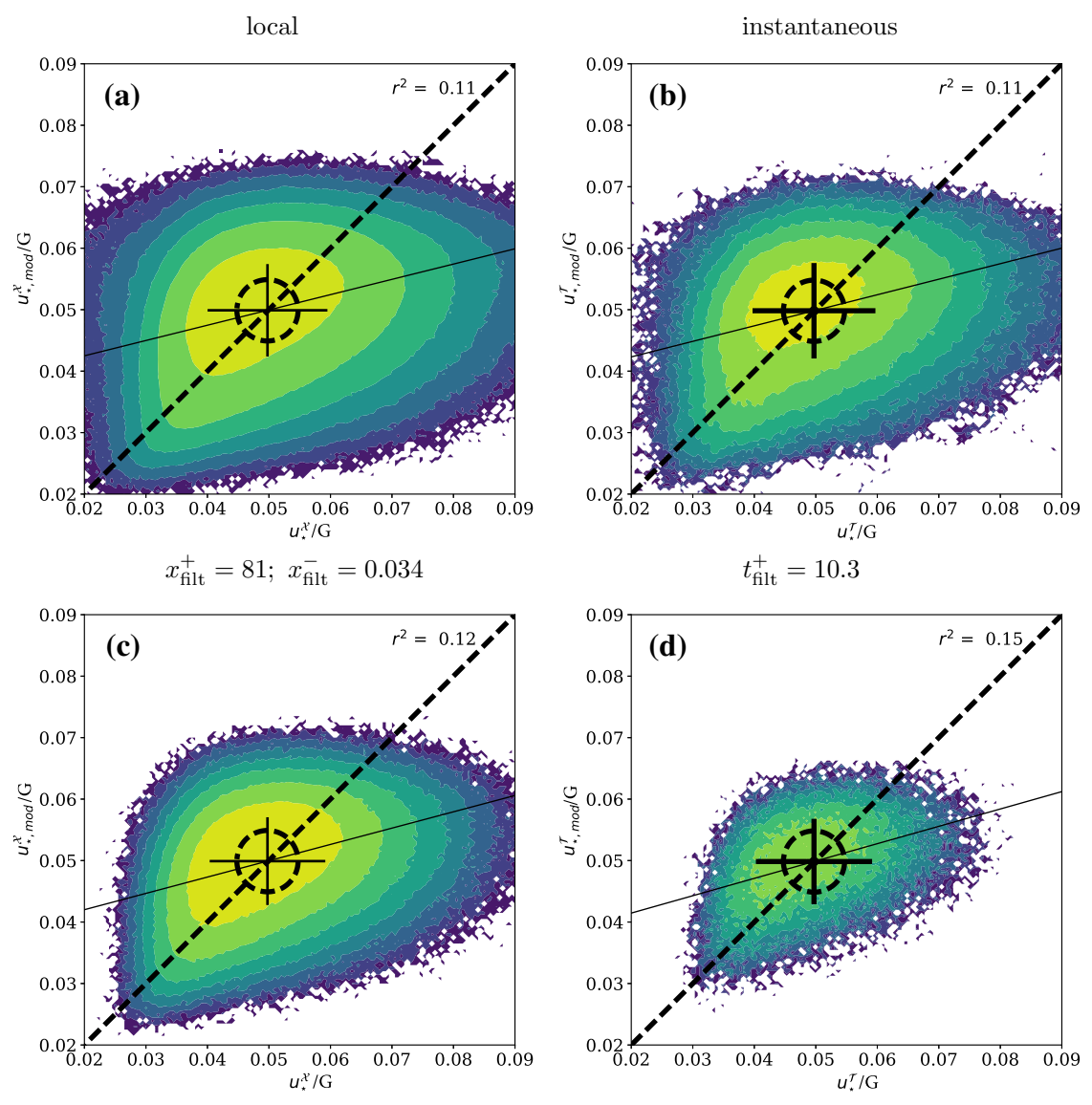

$x_{\text {filt }}^{+}=2600 ; x_{\text {filt }}^{-}=1.10$
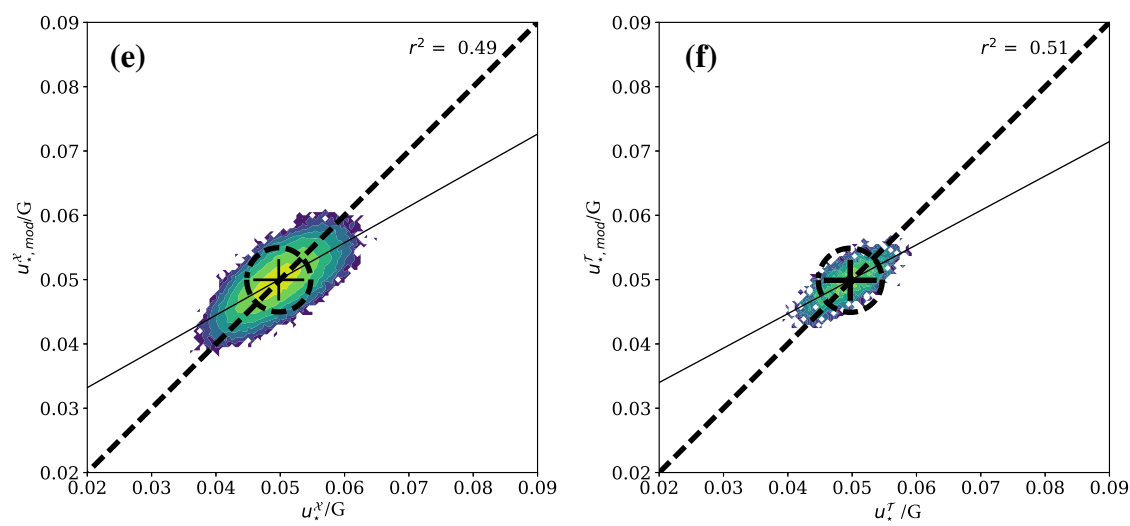

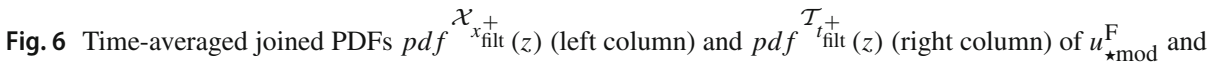
$u_{\star}^{\mathrm{F}}$ for case re_high. Colour scale ranges from white $(0)$ via blue to the respective maximum value in light green. The curve $u_{\star \bmod }^{\mathrm{F}}=u_{\star}^{\mathrm{F}}$ is shown as a thick dashed line. The ensemble average $\left(\left\langle u_{\star}\right\rangle,\left\langle u_{\star \bmod }\right\rangle\right)$ is in the centre of the thick dashed circle and the standard deviation is illustrated by a solid line in the respective direction around the mean. The corresponding least-square fit for the data is shown as a thin solid line, and the corresponding value of $r^{2}$ is given in the top right corner of each panel. Height: $z^{+} \approx 32, z^{-}=0.0135$ 

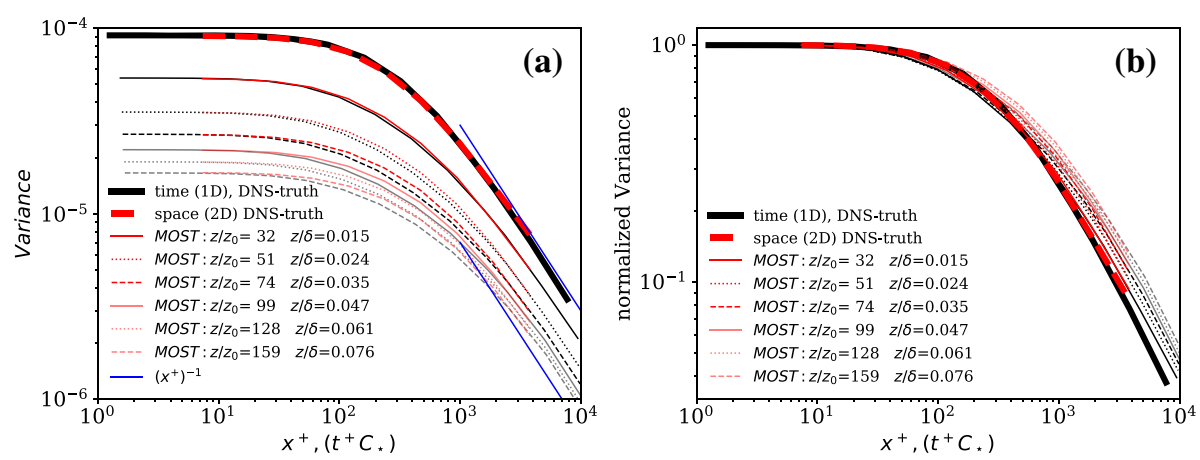

Fig. 7 a Variance of $u_{\star \text { mod }}^{\mathrm{F}}$ at different filter scales for time- and space-averaging at different heights. The averaging time $t^{+}$is multiplied by the advection which is taken into account by the convection-like velocity $C_{\star}$ mapping $t^{+}$to a spatial separation. $\mathbf{b}$ Same as in $\mathbf{a}$, but normalized with the total variance of instantaneous/local data at each height

the joint PDF would collapse onto the line $u_{\star \text { mod }}^{\mathrm{F}}=u_{\star \text { loc }}^{\mathrm{F}}$ regardless of the filter $\mathcal{T}$ or $\mathcal{X}$ and scales $x_{\text {filt }}^{+}$or $t_{\text {filt }}^{+}$used. When considering instantaneous data samples, data do not only scatter substantially (both $u_{\star}$ and $u_{\star \text { mod }}$ vary by about $100 \%$ ), but also the most common value of $u_{\star}$ and $u_{\star \text { mod }}$ does not coincide (maximum of the PDF is not on the 1:1 line). The variance (indicated for both $u_{\star}^{\mathrm{F}}$ and $u_{\star \text { mod }}^{F}$ by the cross centred around the joint mean $\left.\left(\left\langle u_{\star}^{\mathrm{F}}\right\rangle,\left\langle u_{\star, \text { mod }}^{\mathrm{F}}\right\rangle\right)\right)$ in $u_{\star}^{\mathrm{F}}$ is larger than that in the gradient $u_{\star, \bmod }^{\mathrm{F}}$. A linear regression of all pairs $\left(u_{\star}^{\mathrm{F}}, u_{\star, \text { mod }}^{\mathrm{F}}\right)$ shows that the main variation in the data does not adhere to MOST (solid black line) and quantifies the low covariation in terms of $r^{2}=0.11$ for instantaneous data.

When the averaging period or line length increases, the scatter of data converges towards the mean as seen in the middle and lower panels of Fig. 6. Interestingly, this convergence is not only a convergence towards the mean but also a convergence towards the friction prescribed by MOST as is seen by a gradually increasing alignment of the joint PDF of $u_{\star}^{\mathrm{F}}$ and $u_{\star, \text { mod }}^{\mathrm{F}}$ with the line $u_{\star}^{\mathrm{F}}=u_{\star, \bmod }^{\mathrm{F}}$. The increasing alignment is reflected in an increase of $r^{2}$ to up to $\approx 0.5$ for data averaged over 250 viscous time units or several thousand wall units in space.

Qualitatively, these findings also hold for other heights within the ASL, but as a consequence of the larger vertical separation, the coupling between these higher levels and the surface is even weaker and thus the convergence occurs on even longer time or length scales (Fig. 7). Figure 8 illustrates the convergence behaviour at different heights in terms of $p d f^{\mathcal{X}}$. While an increase of the averaging period eventually always yields improved convergence to MOST as long as the level is within the ASL, the rate of convergence decreases when moving away from the surface. The slope of regression fits is far less than unity and the explained variance even at the largest scales does not exceed $20 \%$. This is a consequence of increased eddy size in the turbulent flow at higher levels, and it is consistent with Townsend's attached-eddy hypothesis.

Despite the large aspect ratio $\left(S_{x y}^{+}=\mathcal{O}\left(10^{4}\right)\right.$, cf. Table 2) employed here, there is no convergence towards MOST in a strict sense, even at the largest scale separations available within this study: only about $50 \%$ of the variance is explained by the similarity theory and the slope of the regression fit is far less than unity. This suggests additional mechanisms at work here; indeed, the periodic variation of the wind speed as a consequence of the inertial oscillation is not found in the wall shear stress that is consistent with recent numerical and analytical investigations in similar flow configurations (Shapiro et al. 2016; Fedorovich et al. 

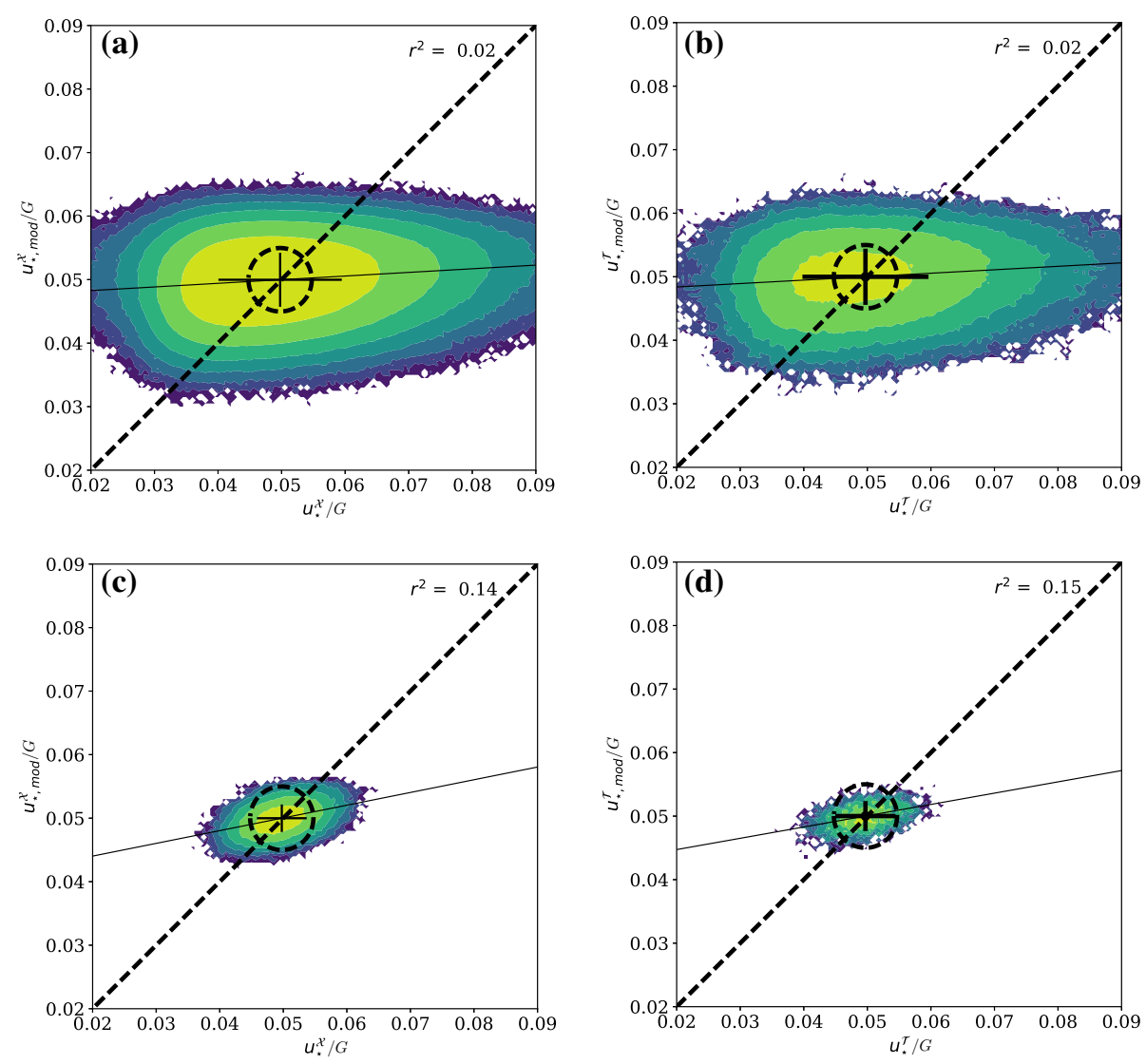

Fig. 8 Convergence for neutral stratification at the far upper end of the logarithmic layer $\left(z^{+} \approx 610, z^{-} \approx\right.$ 0.23 ); otherwise as Fig. 6

2017). Absence of convergence in space-filtered data (not affected by the inertial oscillation) further suggests that the variations in surface-atmosphere coupling due to coherent largescale motions are also not fully captured by MOST. Furthermore, a distinct three-dimensional structure of individual turbulence elements (for instance, a spanwise tilt of those elements) prohibits the full convergence of data that is only filtered along the streamwise direction as shown in Sect. 4.3.

\subsection{Scale Dependence of Variance and the Taylor Hypothesis on Frozen Turbulence}

The most striking property of the friction estimated through MOST is that the variance in $u_{\star \mathrm{mod}}^{\mathrm{F}}$ is too small when compared with the actual variance of the surface friction velocity $u_{\star}^{\mathrm{F}}$. This becomes evident in terms of the slope of $u_{\star \bmod }\left(u_{\star}^{\mathrm{F}}\right)$ which is smaller than unity in all cases (cf. Fig. 6). The underestimation of variance is - almost irrespective of the filter type and scale- $\mathrm{a}$ function of the height from which the friction is estimated. As a consequence of the strict monotony of $\tilde{u}_{\star}(F[u], F[b])$ as a functionof $F[u]$ for $F[b]=0$ (cf. Eq. $7 \mathrm{~b}$ for 
neutral conditions), this relates directly to the decrease of the variance of $u$ (and hence $F[u]$ ) across the logarithmic layer, a well-known property of the ASL (Jiménez 2012; Marusic et al. 2013).

To quantify by how much the variance is underestimated and how this depends on the range of scales considered, we discuss now how the variance of $u_{\star \text {, mod }}^{\mathrm{F}}$ behaves as a function of filter scale for $F=\mathcal{X}_{x^{+}}$and $F=\mathcal{T}_{t^{+}}$(Fig. 7) for the largest, smallest and intermediate scales.

At the instantaneous end of the spectrum of motions $\left(x^{+}, t^{+} \rightarrow 0\right)$, the variance is independent of whether it is estimated from temporally or spatially-resolved data-it is commensurate with the total variance. The convergence occurs at a much faster pace in absolute viscous time units $\left(t^{+}\right)$than it does in viscous space units $\left(x^{+}\right)$as is evident from Fig. 7a; this is already seen from the discrepancy between $t^{+}$and $x^{+}$in Fig. 6. This preferred convergence of fixed-in-time measurements is due to the low turbulence intensity and can be corrected for by a convection-like velocity scale. To match the variance between the time and space dimensions for small turbulent scales, the Taylor hypothesis of frozen turbulence requires that this convection velocity be the local mean velocity, which is indeed the case for the data presented in Fig. 7 (corresponding normalization is not shown).

For the largest filter scales $\left(x^{+} \gtrsim 10^{3}\right)$, the variance depends, irrespective of height, exactly inversely on the filter scales which indicates coherent motions do not affect the convergence at these scales of motion. That is, a purely statistical convergence is observed at these largest scales. An interesting property is the magnitude of the advection velocity for the large-scale patterns labelled here as $C_{\star}$. It can be estimated visually from matching the distribution of variance over space and time (Fig. 7a). Linear dependence of $C_{\star}$ on $U^{+}$with

$$
C_{\star}^{+}\left(z^{+}\right)=0.22 U^{+}\left(z^{+}\right)+6.21
$$

is found across the logarithmic layer $\left(13<U^{+}<19\right.$ corresponding to $30<z^{+}<300$; Fig. 5). There is no physical mechanism known to the author that would require $C_{\star}^{+}$to depend linearly on $U^{+}$; furthermore, coefficients might depend on $R e$ and the geometry of the flow. But, the fact that the appropriate advection velocity at a level $z^{+}$is not $U^{+}\left(z^{+}\right)$reflects the non-locality of turbulence in the inertial layer. This means that the convection velocity for large-scale patterns (as estimated from Taylor's frozen-turbulence hypothesis from matching scale-dependent variances in time and space as in Fig. 7a, b) is different from the mean velocity; in fact it will always be lower than the mean wind speed in the logarithmic layer, provided the bounds for $z^{+}$and $U^{+}$given above are valid. Convection of patterns at a wind speed smaller than $U^{+}(z)$ is consistent with the origin of turbulent structures in the buffer layer and their upward propagation (ejection) into the overlap region of the inner and outer layers. At the surface, where $U^{+}=0, C^{+}\left(z^{+}\right)$does not approach zero, but it is $\left.C_{\star}^{+}\right|_{z=0} \approx 6.5$ (as estimated from the matching between $u_{\star}^{\mathcal{X}}$ and $u_{\star}^{\mathcal{T}}$ at $z=0$ ), consistent with the offset of 6.2 in Eq. 11. This means the appropriate convection velocity for patterns of the surface variance is determined by the advection in the buffer layer; there, $U^{+}$acquires values in the range of $5-10$.

When the variance is normalized by the instantaneous reference (Fig. 7b), a much better collapse of the data for small filter scales is observed. With increasing height, a slower decay of variance emerges at large scales reflecting the increase of the dominant length scale (mixing length) with $\kappa z$, another illustration of consistency of these data with surface-layer theory. At intermediate scales (around $x^{+} \approx 100$ ), the variance of time-filtered estimates decreases faster than that of space-filtered ones. Smaller variance along the temporal vs. the streamwise axis is illustrative of coherent motions that are anisotropic across these dimensions with 

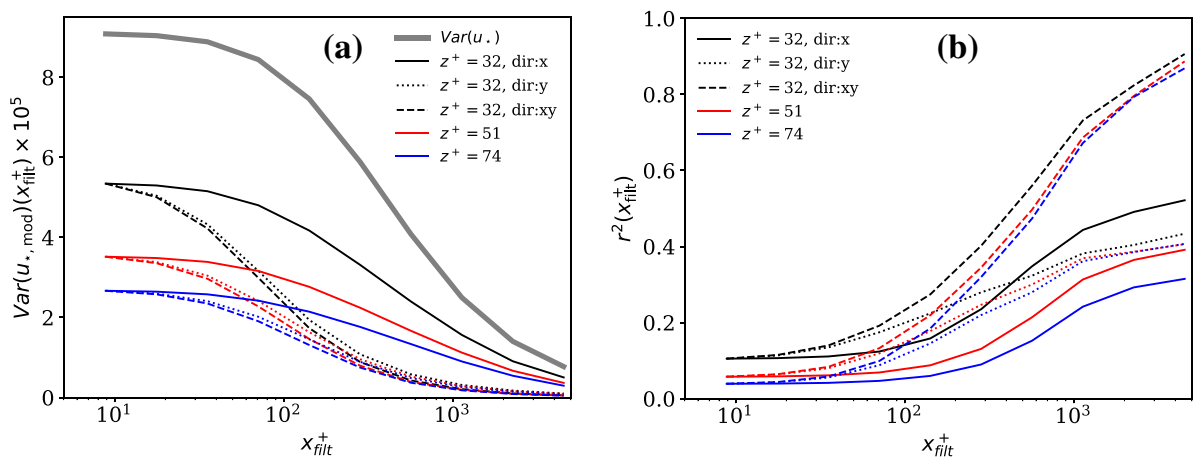

Fig. 9 Scale dependence of variance (panel a) and correlation coefficient $r^{2}$ (panel b) as a function of the filter scale for streamwise ( $O x$, solid), spanwise $(O y$, dotted) and two-dimensional in-plane $(O x y$, dashed) filtering. Colours code height above the surface (black: $y^{+}=32$; red: $y^{+}=51$; blue: $y^{+}=74$ ) and line styles the direction or directions along which filtering was applied

stronger persistence in the streamwise direction than in time. This is particularly noteworthy as in Fig. 7b the large-scale advection is already accounted for by $C_{\star}$.

\subsection{Convergence in Horizontal Planes}

When simulating flows in the ASL, filters are often applied that operate isotropically along at least the horizontal dimensions (different filter properties or length scales are often used in the vertical direction to account for large variability in background profiles). Using the most common filter operator applied in LES, the average over a box of finite size, this corresponds to an averaging over square parts of the domain also referred to as coarse graining (cf. Sect. 2.4). The characteristic feature of shear turbulence in the vicinity of a surface is streamwise elongated streaks characterized by a relatively strong persistence along $O x$. The large aspect ratio of surface streaks together with their persistence suggests a considerably increased variability along the flow-lateral direction $O y$ when compared to $O x$. This implies a larger number of streaks per unit length along $O y$ and thus faster convergence when filtering along either the $O y$ alone or over horizontal boxes $O x y$. Indeed, the variance in estimated friction decreases much faster as a function of the averaging scale when filtering the data along $O y$ (dashed vs. dotted lines in Fig. 9a). The difference is so large that averaging along Oxy instead of lines does not substantially improve the convergence. That means, in terms of the variance (which is commensurate with the sampling uncertainty of the measurement), the filter over a horizontal box of size $l_{0} \times l_{0}$ is well approximated by that along flow-lateral lines of length $l_{0}$.

The convergence properties in pdf space differ between $p d f^{\mathcal{X}}(z)$ and $p d f^{\mathcal{Y}}(z)$ vs. $p d f^{\mathcal{X Y}}(z)$. The correlation between $u_{\star \text { mod }}^{\mathcal{X}}$ and $u_{\star \text { loc }}^{\mathcal{X}}$ indicated by $r^{2}$ (Fig. 9b) not only grows faster as a function of the averaging scale, but it also saturates at larger values; indeed, the possibility of convergence towards $r_{\mathcal{X} Y}^{2}=1$ in the limit of large averaging scales cannot be excluded for filtering along horizontal planes $O x y$. On the contrary, the correlation of spanwise averaged data suggests that $r_{\mathcal{Y}}^{2}<1$ as $x_{\text {filt }}$ becomes large. This behaviour underlines the three-dimensional character of the turbulence in Ekman flow, even in the lowest part of the ASL. There is a systematic displacement of structures in the lowest part of the ASL along both horizontal directions which may only be covered by a two-dimensional filter. 

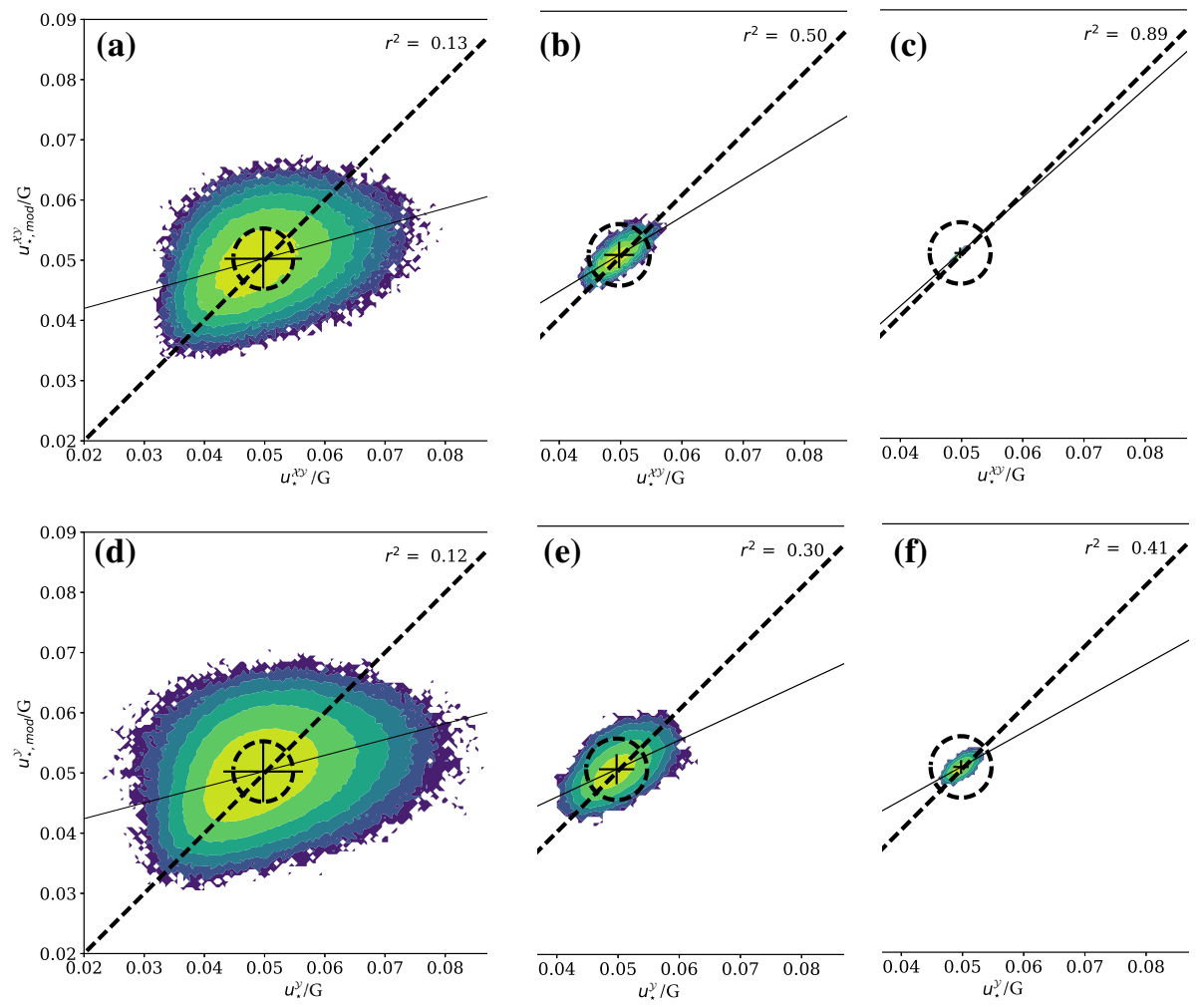

Fig. $10 p d f^{\mathcal{X} Y}$ (upper row) and $p d f^{\mathcal{Y}}$ (lower row) shown as in Fig. 6. The filter length scales are $x_{\text {filt }}^{+}=60$ (left column) $x_{\text {filt }}^{+}=480$ (center column) and $x_{\text {filt }}^{+}=3800$ (right column)

The question remains whether this improved correlation of between $u_{\star \text { mod }}^{\mathcal{X}}$ and $u_{\star}^{\mathcal{X Y}}$ is also a convergence towards the exact prediction by MOST as one might obtain perfect correlation with a line of arbitrary slope. The increased correlation for the two-dimensional horizontally filtered data $\left(r^{2}=0.89\right.$ for the filter $\mathcal{X} \mathcal{Y}$ in Fig. $10 \mathrm{c}$ vs. $r^{2}=0.41$ for the filter $\mathcal{Y}$ in Fig. 10f) goes hand in hand with an increased slope of the least-square fit towards the theoretical limit of unity (Fig. 10a-c). For one-dimensionally filtered data, the limit of convergence is constrained by systematic vertical misalignment of turbulent structures, and this is independent of whether averages are taken along the streamwise, spanwise or temporal dimension. Applying Taylor's hypothesis, this vertical misalignment must affect the time-filtered data in the same way as it affects the streamwise-filtered data. In conclusion, convergence towards MOST in a three-dimensionally turbulent flow may only be strictly observed for horizontally filtered data.

\section{Convergence to MOST in Stable Conditions}

An important merit of MOST is the estimation of the stability effect on profiles of velocity and buoyancy (or the temperature in an observational context). Beyond investigation of the mere adherence to logarithmic scaling in the bulk and associated convergence behaviour discussed 


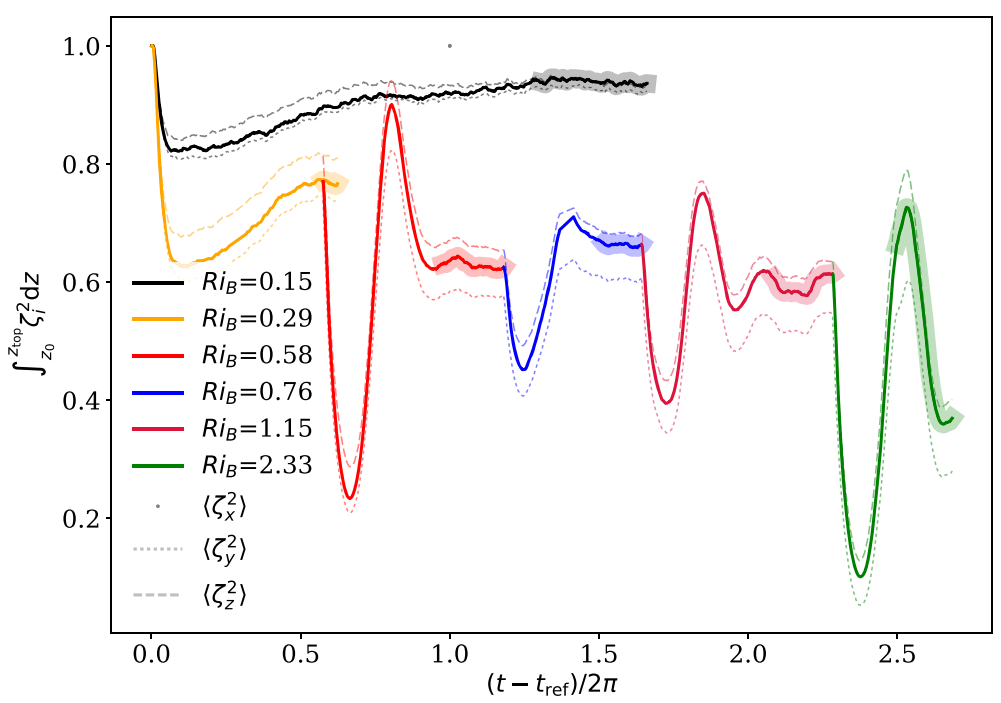

Fig. 11 Root-mean square of the three components of vorticity integrated over the ASL. The analysis period used henceforth is confined to the quasi-steady periods marked by a shading around the streamwise component of vorticity $\left\langle\zeta_{x}^{2}\right\rangle$

above, we turn now to this stratified case where the stability correction $\zeta$ enters similarity theory as a parameter. The desirable property of statistical steadiness, conveniently exploited in Sect. 4, is lost once Ekman flow is exposed to stratification: the statistical description of the flow becomes a function of the time besides its dependence on the bulk stratification characterized by $R i$ (Sect. 2.2). Hence, the convergence analysis is constrained to spatially-resolved data. With respect to the convergence in the time domain, a rigorous analysis of convergence is not possible absent modifications of the set-up compensating for time dependence of the surface buoyancy flux.

While there does not exist a truly steady state of the present set-up once the stratification becomes finite, the stratified cases vary on different time scales and acquire a quasi-steady state after an initial transition (Ansorge and Mellado 2014). In Fig. 11, the turbulence state is illustrated by the vertically integrated vorticity fluctuations. This shows an initial adaptation to the reduced surface buoyancy, in which the production in the buffer layer is cut. The subsequent increase enters into a strongly damped oscillation and finally exhibits a quasi-steady behaviour. We investigate here only this quasi-steady period (marked by opaque shading in Fig. 11), in which the turbulence has acquired a quasi-equilibrium with respect to the surface boundary condition.

\subsection{Bulk Similarity}

Broad consensus exists regarding the applicability of similarity theory to bulk averages in stratified conditions as long as the non-dimensional stratification expressed by a flux Richardson number or the Obukhov parameter $\zeta \equiv z / L$ does not become too large (Foken 2006; Grachev et al. 2012; Optis et al. 2014, 2016). The stability correction function estimated from the present DNS data (Fig. 12) agrees with findings from previous simulation studies (van de Wiel et al. 2008; Shah and Bou-Zeid 2014; Ansorge and Mellado 2014) and also 

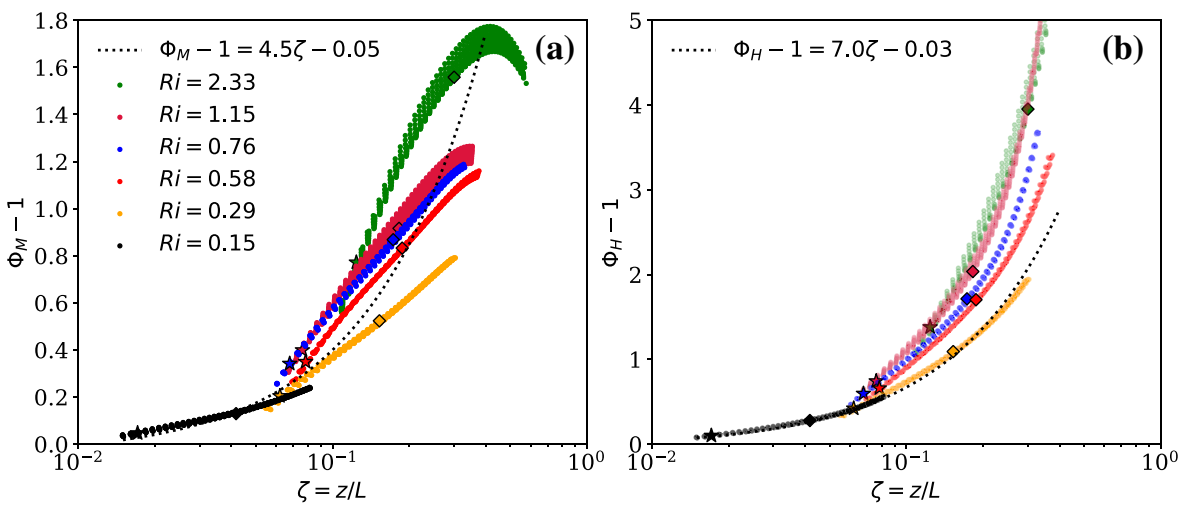

Fig. 12 Stability correction estimated as outlined in Eq. 3e for momentum (a) and buoyancy (b). Here, data are shown in the logarithmic layer $\left(30<z^{+}<0.1 \delta^{+}\right.$, where $\delta^{+}=u_{\star}^{2} /(v f)$ is the boundary-layer thickness $\delta \equiv u_{\star} / f$ expressed in wall units) and within the period marked in Fig. 11b. Fixed heights as fraction of the boundary-layer thickness $\delta \equiv u_{\star} / f$ are marked by an asterisk $(\delta=0.02)$ and a diamond $(\delta=0.05)$

recent observational fits (Högström 1996; Foken 2006), despite its limits in terms of an intermediate Reynolds number and a constrained physical set-up. While agreement is very good at weak stratification and for small $z$ (left end of the curves in Fig. 12), the stability correction estimated from the DNS data already deviates from a linear fit by a distinct right curvature at $\zeta \approx 0.1$; this is relatively early in comparison with observational data, where the stability correction saturates around $\zeta_{\kappa}=1(\zeta=0.4)$ at $\Phi_{M}-1=6 \kappa \approx 2.5$ [Brutsaert (2005) after Businger et al. (1971) with correction by Högström (1988)], and it may be associated with the limited extent of the logarithmic region at the intermediate $R e$ employed in the present simulations. The estimated stability correction for momentum is further affected by increased turning of the wind in stable conditions which is not taken into account by MOST as it only considers the surface-shear-aligned velocity component. The actual importance of this wind turning for the stability correction of momentum is underlined by the much better adherence of the stability correction for buoyancy to the estimation based on MOST.

In the present bulk analysis (cf. Fig. 12) three parameters, namely stratification, height, and time are varied. This is in contrast to most observational studies, but also many assessments based on numerically generated data, where-due to the practicability constraints imposed in field work - data are commonly considered at a fixed height. This triple variation constitutes a more rigorous test of the generality of the non-dimensional formulation than variation of only one parameter, and also illustrates the limits of applicability. The data in Fig. 12 are stratified in colour by $R i_{B}$ and the scatter plots show profiles at each time instant within the time span marked in Fig. 11b. For each curve, the main variation from left to right is the variation of height $z$. Another dimension along which the data vary here is time as the surface flux is free to evolve (as a consequence of the Dirichlet boundary condition, cf. Sect. 2.2) and decreases in magnitude over time as the flow adapts more and more to the imposed surface boundary condition. In particular those cases with very strong stable stratification (beyond $R i_{B} \approx 0.76$ ) exhibit a substantial variation of the stability correction over time at fixed $\zeta$ which illustrates that processes like inertial oscillations and other out-of-equilibrium dynamics are at work here that are not covered by MOST.

In the stratified cases with $R i>0.29$, the stability parameter $\zeta$ no longer collapses the data. These cases are fraught with complex dynamics that arise with the partial collapse of turbulence in response to increasing density stratification, i.e. global intermittency (cf. 
Ansorge and Mellado 2014; Shah and Bou-Zeid 2014; Deusebio et al. 2015). Intermittent turbulence is not appropriately characterized by bulk and conventional statistics; instead, conditional sampling can be employed to gain insight into the complex dynamics of such intermittent flows (Ansorge and Mellado 2016; Ansorge 2016). Even in critical conditions (at intermediate stratification with very little or no global intermittency), $L$ alone does not characterize the flow state appropriately (Hooijdonk et al. 2018). In these cases, the buoyancy flux (and thus $L$ ) is limited while the bulk stratification and also the wind speed correction increase further, which is consistently observed for both the stability correction of buoyancy and momentum and thus does not appear to impact on the turbulent Prandtl number. Nonetheless, an investigation of the convergence behaviour towards the mean is enlightening as systematic scatter can illustrate whether there is a scale of motion beyond or below which the similarity theory is applicable in principle or not, irrespective of an exact collapse of the mean properties.

\subsection{Convergence and Scale Dependence}

We now investigate as an example the convergence properties for two stratified cases with $R i_{B}=0.29$ and $R i_{B}=0.76$. Being super-critical in terms of the bulk stratification (Fig. 12 and Sect. 5.1), these cases are in a spatio-temporally intermittent state, i.e. the flow is not turbulent throughout but there are non-turbulent patches stretching vertically across the ASL (Ansorge and Mellado 2016). The fraction of the non-turbulent patches increases with stratification but is still on the order of $10 \%$ for both cases. These non-turbulent fractions are associated with very small values of the surface friction and explain the increasing fraction of the joint PDF at small friction values compared to the neutral reference-both modelled and estimated. Apart from this extension towards low values of friction, the joint PDF $p d f^{\mathcal{X} Y}$ of the instantaneous data is comparable in terms of both its shape and its first and second moments with that in neutral conditions at a similar height (Fig. 13). While the overall shape of the distribution does not change, the variance increases slightly, and the correlation coefficient between the friction estimated by similarity theory and the actual friction becomes exactly zero. In contrast to neutral stratification, where the similarity theory explains a sufficient share of the variance as the filter scale increases, the opposite is the case in stable conditions: while the averages of the estimated friction are still within 5-10\% of the actual friction, the scatter of locally-averaged data is not governed by MOST. In fact, the discrepancy increases as the filter scale is increased illustrating a decoupling of variability in the ASL from the actual surface friction while the means are still approximately constrained by MOST. For the more stable case with $R i_{B}=0.76$, the $r^{2}$ value increases as a consequence of negative correlation, which means the MOST-estimated friction velocity is actually decreasing with increasing surface friction as seen by the negative slope of the least-squares fit in Fig. 13.

This negative correlation between estimates of the similarity theory for the spatiallyfiltered local friction and the actual friction has two physical reasons. First, intermittency acts to decrease the surface friction, but, in the absence of turbulence, lower friction causes higher wind speed away from the surface. Second, even in conditions that are turbulentthroughout, frictional decoupling occurs in stratified rotating atmospheric flows (Fedorovich and Shapiro 2017). Such frictional decoupling is hypothesized to be associated with the wind speed maxima found in low-level jets (Parish and Oolman 2010). 

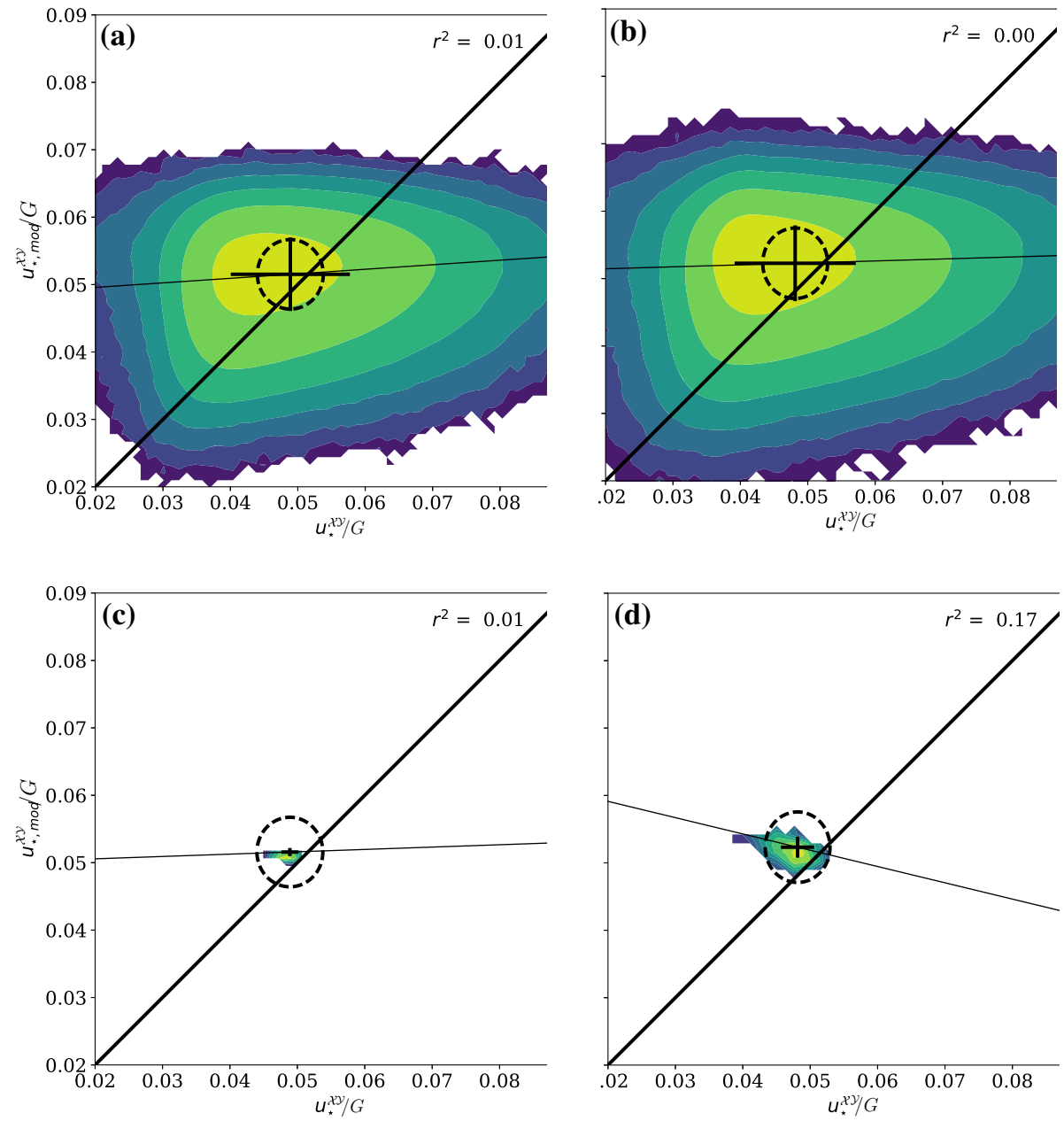

Fig. 13 Joint PDFs $\left.p d f \mathcal{X Y Y}_{\left(z^{+}\right.} \approx 50\right)$ of $u_{\star \operatorname{Mod}}^{\mathcal{X}}$ and $u_{\star}^{\mathcal{X} \mathcal{Y}}$ varying Richardson number and averaging box size as indicated by figure labels; otherwise as Fig. 6

\section{Discussion and Conclusions}

The scale dependence of surface-atmosphere coupling is investigated using DNS of a prototypical PBL, namely Ekman flow over a smooth surface under both neutral and stable stratification. The coupling is quantified in terms of the propagation of variances through the lowest part of the atmosphere and compared to predictions by Monin-Obukhov similarity theory (MOST), a closure employed in virtually all numerical models of atmospheric flows. Surface friction is expressed as a function of wind speed, buoyancy, and the height in the atmospheric surface layer (ASL) alone by rigorous inversion of the similarity function. Hence, comparison of estimated and actual surface friction is free of self-correlation. In all cases considered here, box-averaged simulation data agree with the similarity theory within its well-known limits in terms of height and stratification strength. As a consequence of the imposed spatial homogeneity, the variance in between samples must approach zero 
if the averaging scale of the individual samples increases; this is indeed the case: variance converges exactly inversely with filter length scale in the limit of the largest filter scalesindicative of the absence of long-term memory at these scales in the turbulent flow under consideration. A note of caution is raised with regards to the local application of similarity theory: the modelled and actual friction differ both regarding the convergence towards the large-scale limit and the variance of instantaneous data.

The variance of surface friction velocity is underestimated by MOST_-with immediate implication for the surface momentum flux, a key parameter in defining the turbulent state of the PBL. This occurs in all configurations investigated, but the effect is smaller for smaller heights and larger filter scale. Depending on the way in which MOST is applied, the error has opposing effects: applying MOST to estimate wind speed in the ASL as a function of the surface friction velocity overestimates the variance thus adding an artificial source of turbulence and hence diffusion; the propagation of surface patterns into the atmosphere is thereby erroneously enhanced. Applying MOST to estimate the friction velocity at the surface will underestimate the variance thus damping the impact of atmospheric variability on the surface parameters; the propagation of atmospheric variance patterns to the surface is thereby erroneously damped. This asymmetry of the error introduced by local application of MOST is particularly important for studies employing different kinds of boundary conditions to study a problem as it will add up for such investigations.

Beyond the peculiar convergence behaviour of data towards the mean, a strong dependence on the sampling direction is found. The large anisotropy of near-surface streaks leads to a slower convergence of data that are filtered along the direction of the wall shear stress in comparison to data filtered along the flow-lateral direction. For both filtering approaches, the variation of filtered data, even at the largest scales, is not governed by similarity theory, but there is a bias towards lower modelled friction velocity for high wind speed and higher modelled friction velocity for low wind speed. Also the scatter is not governed by similarity theory to a large extent, which is best quantified in terms of the correlation between the modelled and actual stress for increasing filter sizes that saturates around $r^{2}=0.5$, a rather small value given that MOST is the only model used to link the two. If, however, box averages along both horizontal dimensions are used, correlations close to unity are obtained and the friction velocity modelled based on ASL wind speed agrees almost perfectly with the actual surface friction velocity for filter sizes beyond several thousand wall units. The better convergence of samples when two-dimensional horizontal averages are used instead of one-dimensional ones hints at the three-dimensional structure of the actual turbulence elements that are crucial in coupling the surface and the atmosphere. These elements must in fact have a preferred vertical misalignment for such convergence and correlation properties to be obtained. It remains to be found whether this three-dimensional behaviour is a distinct property of Ekman flow where the stress and shear tensor are misaligned as a consequence of rotation, or if it can also be found in simpler flow configurations.

In stable conditions, the bulk profiles agree with similarity theory until the maximum buoyancy flux is reached and the turbulence becomes intermittent. Accounting for non-stationarity, convergence is only considered in the spatial domain and only for two-dimensional horizontal box averages when these stratified cases are considered. Due to the turbulence intermittency and a frictional decoupling associated with the inertial oscillation, similarity theory does not govern at any scale the joint fluctuation of wind speed in the lower part of the ASL and friction at the surface. In fact, the skill of similarity theory in predicting local variations decreases (and eventually becomes negative) with increasing averaging scale while the mean values are still constrained to within $10 \%$. Hence, in strongly stratified conditions, similarity theory should be applied to filtered quantities rather than locally. 
Data are given here in both inner (wall) and outer (geostrophic) scalings. Application or transfer of the present results to flows at geophysical scale separation requires answering both the question as to in which of the aforementioned scalings do the results presented here become independent of the Reynolds number, and to address the role of roughness. Regarding $R e$, no definite answer on the dependence of the scalings is possible. The dominant role of the wall in these processes, however, suggests that wall-scaling is appropriate. Assuming so yields transition scales on the order of 100 to 1000 wall units - still relatively small compared to the ones used in modern general circulation models, while recent LES approaches actually reach grid resolution below these scales. Regarding roughness, the analogy of the roughness scale to the viscous units may be applied over relatively small roughness elements [smaller than 3-5 wall units (Monin 1970)]. The logarithmic scaling over a smooth wall suggests that the corresponding reference scale for a rough flow is $z_{\text {rough }}^{+} \approx 0.1$. In terms of the roughness length scale, the required averaging scale is hence one order of magnitude larger than in terms of the wall unit. Even when assuming a roughness scale $z_{\text {rough }}=0.01 \mathrm{~m}$, this implies an averaging scale of several tens of metres.

Acknowledgements We wish to acknowledge insightful discussion on the topic with Yaping Shao, Evgeni Fedorovich, Elie Bou-Zeid and Juan Pedro Mellado. We further thank Bas van de Wiel and two other anonymous reviewers for their helpful and constructive comments on the manuscript. This work was funded by the German Research Council (DFG) through the Transregional Research Collaborative \#32 Patterns in LandSurface-Atmosphere Systems (TR32). Computing time was provided through the Projects HHH0 7 and HKU2 4 by the Jülich Supercomputing Centre. Financial support through a UoC PostDoc Grant by the University of Cologne as part of its institutional strategy for the support of early-stage researchers within the excellence initiative is gratefully acknowledged.

Open Access This article is distributed under the terms of the Creative Commons Attribution 4.0 International License (http://creativecommons.org/licenses/by/4.0/), which permits unrestricted use, distribution, and reproduction in any medium, provided you give appropriate credit to the original author(s) and the source, provide a link to the Creative Commons license, and indicate if changes were made.

\section{Appendix 1: Estimating Surface Friction Velocity and Buoyancy Through MOST}

$U^{+}, z^{+}$and $L$ involve the surface fluxes. Hence, estimation of $u_{\star}$ loc and $b_{\star}$ loc requires solving the following non-linear set of equations for $u_{\star}$ loc and $b_{\star \text { loc }}$ as a function of $z, U(z)$ and $B(z)$ For $u_{\star}$ loc $>0$ and $b_{\star \text { loc }}>0$, i.e. in stably stratified conditions:

$$
\begin{aligned}
& \kappa U=u_{\star \mathrm{loc}} \ln \left(c_{z} u_{\star \mathrm{loc}}\right)+c_{M} \frac{b_{\star \mathrm{loc}}}{u_{\star \mathrm{loc}}}, \\
& \kappa B=b_{\star \mathrm{loc}} \ln \left(c_{z} u_{\star \mathrm{loc}}\right)+c_{H} \frac{b_{\star \mathrm{loc}}^{2}}{u_{\star \mathrm{loc}}^{2}},
\end{aligned}
$$

with $c_{z}=z / 8 v, c_{M}=\beta_{M}\left(z-z_{0}\right)$, and $c_{H}=\beta_{H}\left(z-z_{0}\right)$. Elimination of $b_{\star l o c}$ and letting $\Lambda \equiv u_{\star l o c} \ln \left(c_{z} u_{\star l o c}\right)$ yields

$$
\Rightarrow c_{H}[\kappa U-\Lambda]^{2}=c_{M}\left\{\kappa c_{M} B-\Lambda[\kappa U-\Lambda]\right\} .
$$

Depending on $c_{H}-c_{M}$, Eq. $12 \mathrm{~b}$ has one or two solutions for $\Lambda$ :

$$
\tilde{\Lambda}(U, B)= \begin{cases}\frac{\kappa U^{2}-B c_{M}}{U} & \text { if } c_{H}=c_{M} \\ \frac{\kappa U\left(2 c_{H}-c_{M}\right) \pm c_{M} \sqrt{\kappa} \sqrt{4 B\left(c_{H}-c_{M}\right)+\kappa U^{2}}}{2\left(c_{H}-c_{M}\right)} & \text { else. }\end{cases}
$$


The solution for $c_{H} \neq c_{M}$ with positive sign in front of the radicand is disregarded as unphysical by matching the solution to its neutral limit $\left(\partial_{z} B=0 ; b_{\star}=0\right)$ and obtain

$$
\begin{aligned}
& \tilde{u}_{\star}(U, B)=\frac{\tilde{\Lambda}}{W\left(c_{z} \tilde{\Lambda}\right)}, \\
& \tilde{b}_{\star}(U, B)=\tilde{\Lambda} \frac{\kappa U-\tilde{\Lambda}}{c_{M} W\left(c_{z} \tilde{\Lambda}\right)},
\end{aligned}
$$

with $W$ the product logarithm.

\section{References}

Adrian RJ (2007) Hairpin vortex organization in wall turbulence. Phys Fluids 19(041):301

Alfredsson PH, Johansson AV, Haritonidis JH, Eckelmann H (1988) The fluctuating wall-shear stress and the velocity-field in the viscous sublayer. Phys Fluids 31(5):1026-1033

Aluie H, Kurien S (2011) Joint downscale fluxes of energy and potential enstrophy in rotating stratified Boussinesq flows. Europhys Lett 96(4):44,006

Ament F, Simmer C (2006) Improved representation of land-surface heterogeneity in a non-hydrostatic numerical weather prediction model. Boundary-Layer Meteorol 121(1):153-174

Ansorge C (2016) Analyses of turbulence in the neutrally and stably stratified planetary boundary layer. Springer Theses, Springer, Dordrecht

Ansorge C, Mellado JP (2014) Global intermittency and collapsing turbulence in the stratified planetary boundary layer. Boundary-Layer Meteorol 153(1):89-116

Ansorge C, Mellado JP (2016) Analyses of external and global intermittency in the logarithmic layer of Ekman flow. J Fluid Mech 805:611-635

Avissar R, Pielke RA (1989) A parameterization of heterogeneous land surfaces for atmospheric numericalmodels and its impact on regional meteorology. Mon Weather Rev 117(10):2113-2136

Brutsaert W (2005) Hydrology: an introduction. Cambridge University Press, Cambridge

Businger JA, Yaglom AM (1971) Introduction to Obukhov's paper on 'turbulence in an atmosphere with a non-uniform temperature'. Boundary-Layer Meteorol 2(1):3-6

Businger JA, Wyngaard JC, Izumi Y, Bradley EF (1971) Flux-profile relationships in the atmospheric surface layer. J Atmos Sci 28:181-189

Charnock H (1955) Wind stress on a water surface. Q J R Meteorol Soc 81(350):639-640

Claussen M (1991) Estimation of areally-averaged surface fluxes. Boundary-Layer Meteorol 54(4):387-410

Coleman GN, Ferziger JH, Spalart PR (1990) A numerical study of the turbulent Ekman layer. J Fluid Mech 213:313-348

Deusebio E, Caulfield CP, Taylor JR (2015) The intermittency boundary in stratified plane Couette flow. J Fluid Mech 781:298-329

Fairall CW, Bradley EF, Hare JE, Grachev AA, Edson JB (2003) Bulk parameterization of air-sea fluxes: updates and verification for the COARE algorithm. J Clim 16(4):571-591

Fedorovich E, Gibbs JA, Shapiro A (2017) Numerical study of nocturnal low-level jets over gently sloping terrain. J Atmos Sci 74:2813-2834

Fedorovich E, Shapiro A (2017) Oscillations in Prandtl slope flow started from rest. Q J R Meteorol Soc 143(703):670-677

Foken T (2002) Some aspects of the viscous sublayer. Meteorol Z 11(4):267-272

Foken T (2006) 50 Years of the Monin-Obukhov similarity theory. Boundary-Layer Meteorol 119(3):431-447

Foken T, Kitajgorodskij SA, Kuznecov OA (1978) On the dynamics of the molecular temperature boundary layer above the sea. Boundary-Layer Meteorol 15(3):289-300

Garratt J (1990) The internal boundary layer: a review. Boundary-Layer Meteorol 50(1-4):171-203

Giorgi F, Avissar R (1997) Representation of heterogeneity effects in Earth system modeling: experience from land surface modeling. Rev Geophys 35(4):413-437

Grachev AA, Andreas EL, Fairall CW, Guest PS, Persson POG (2012) The critical Richardson number and limits of applicability of local similarity theory in the stable boundary layer. Boundary-Layer Meteorol 147(1):51-82

Gryning SE, Batchvarova E, Brümmer B, Jørgensen H, Larsen S (2007) On the extension of the wind profile over homogeneous terrain beyond the surface boundary layer. Boundary-Layer Meteorol 124(2):251-268 
Haugen DA, Kaimal JC, Bradley EF (1971) An experimental study of Reynolds stress and heat flux in the atmospheric surface layer. Q J R Meteorol Soc 97(412):168-180

Haugen DA, Izumi Y, Kaimal JC, Wyngaard JC, Coté OR, Caughey SJ, Readings CJ (1976) Turbulence structure in the convective boundary layer. J Atmos Sci 33:2152-2169

Högström U (1988) Non-dimensional wind and temperature profiles in the atmospheric surface layer: a reevaluation. Boundary-Layer Meteorol 42:55-78

Högström U (1996) Review of some basic characteristics of the atmospheric surface layer. Boundary-Layer Meteorol 78(3-4):215-246

Hooijdonk IGS, Clercx HJH, Ansorge C, Moene AF, van de Wiel BJH (2018) Parameters for the collapse of turbulence in the stratified plane Couette flow. J Atmos Sci. https://doi.org/10.1175/JAS-D-17-0335.1

Jiménez J (2012) Cascades in wall-bounded turbulence. Annu Rev Fluid Mech 44(1):27-45

Katsoulakis MA, Plecháč P, Rey-Bellet L (2008a) Numerical and statistical methods for the coarse-graining of many-particle stochastic systems. J Sci Comput 37(1):43-71

Katsoulakis MA, Plecháč P, Rey-Bellet L, Tsagkarogiannis DK (2008b) Mathematical strategies in the coarsegraining of extensive systems: error quantification and adaptivity. J Non-Newton Fluid Mech 152(13):101-112

Kawai S, Larsson J (2012) Wall-modeling in large eddy simulation: length scales, grid resolution, and accuracy. Phys Fluids 24(1):015,105

Krogstad PÅ, Kaspersen JH, Rimestad S (1998) Convection velocities in a turbulent boundary layer. Phys Fluids 10(4):949-10

Li Q, Bou-Zeid E, Vercauteren N, Parlange MB (2018) Signatures of air-wave interactions over a large lake. Boundary-Layer Meteorol 167(3):1-24

Lin CC (1953) On Taylor's hypothesis and the acceleration terms in the Navier-Stokes equations. Q Appl Math 10(4):295-306

Marusic I, Monty JP, Hultmark M, Smits AJ (2013) On the logarithmic region in wall turbulence. J Fluid Mech 716:R3-1-R3-11

Monin AS (1970) The atmospheric boundary layer. Annu Rev Fluid Mech 2:225-250

Moin P (2009) Revisiting Taylor's hypothesis. J Fluid Mech 640:1-4

Moin P, Mahesh K (1998) Direct numerical simulation: a tool in turbulence research. Annu Rev Fluid Mech 30:539-578

Monin AS, Obukhov AM (1954) Basic laws of turbulent mixing in the atmosphere near the ground. Trudy Geofiz Inst AN SSSR 24(151):163-187 (in Russian)

Nieuwstadt FTM (1984) The turbulent structure of the stable, nocturnal boundary layer. J Atmos Sci 41:22022216

Obukhov AM (1946) Turbulence in an atmosphere with inhomogeneous temperature. Trudy Geofiz Inst AN SSSR 1:95-115 (in Russian)

Obukhov AM (1971) Turbulence in an atmosphere with a non-uniform temperature. Boundary-Layer Meteorol 2(1):7-29

Optis M, Monahan A, Bosveld FC (2014) Moving beyond Monin-Obukhov similarity theory in modelling wind-speed profiles in the lower atmospheric boundary layer under stable stratification. Boundary-Layer Meteorol 153(3):497-514

Optis M, Monahan A, Bosveld FC (2016) Limitations and breakdown of Monin-Obukhov similarity theory for wind profile extrapolation under stable stratification. Wind Energy 19(6):1053-1072

Parish TR, Oolman LD (2010) On the role of sloping terrain in the forcing of the Great Plains low-level jet. J Atmos Sci 67(8):2690-2699

Park GI, Moin P (2014) An improved dynamic non-equilibrium wall-model for large eddy simulation. Phys Fluids 26(1):015,108

Sayde C, Thomas CK, Wagner J, Selker J (2015) High-resolution wind speed measurements using actively heated fiber optics. Geophys Res Lett 42(22):10,064-10,073

Shah SK, Bou-Zeid E (2014) Direct numerical simulations of turbulent Ekman layers with increasing static stability: modifications to the bulk structure and second-order statistics. J Fluid Mech 760:494-539

Shao Y, Liu S, Schween JH, Crewell S (2013) Large-eddy atmosphere-land-surface modelling over heterogeneous surfaces: model development and comparison with measurements. Boundary-Layer Meteorol 148(2):333-356

Shapiro A, Fedorovich E, Rahimi S (2016) A unified theory for the Great Plains nocturnal low-level jet. J Atmos Sci 73(8):3037-3057

Simmer C, Thiele-Eich I, Masbou M, Amelung W, Bogena H, Crewell S, Diekkrueger B, Ewert F, Franssen HJH, Huisman JA, Kemna A, Klitzsch N, Kollet S, Langensiepen M, Loehnert U, Rahman ASMM, Rascher U, Schneider K, Schween JH, Shao Y, Shrestha P, Stiebler M, Sulis M, Vanderborght J, Vereecken 
H, van der Kruk J, Waldhoff G, Zerenner T (2015) Monitoring and modeling the terrestrial system from pores to catchments. Bull Am Meteorol Soc 96(10):1765-1787

Spalart PR (1989) Theoretical and numerical studies of a three-dimensional turbulent boundary layer. J Fluid Mech 205:319-340

Spalart PR, Coleman GN, Johnstone R (2008) Direct numerical simulation of the Ekman layer: a step in Reynolds number, and cautious support for a log law with a shifted origin. Phys Fluids 20(10):101,507 (Retracted article. See, vol. 21, art. no. 109901, 2009)

Vereecken H, Pachepsky Y, Simmer C, Rihani J, Kunoth A, Korres W, Graf A, Franssen HJH, Thiele-Eich I, Shao Y (2016) On the role of patterns in understanding the functioning of soil-vegetation-atmosphere systems. J Hydrol 542:63-86

van de Wiel BJH, Moene AF, De Ronde WH, Jonker HJJ (2008) Local similarity in the stable boundary layer and mixing-length approaches: consistency of concepts. Boundary-Layer Meteorol 128(1):103-116

Wills JAB (1964) On convection velocities in turbulent shear flows. J Fluid Mech 20(3):417-432

Zilitinkevich SS, Esau IN (2007) Similarity theory and calculation of turbulent fluxes at the surface for the stably stratified atmospheric boundary layer. Boundary-Layer Meteorol 125(2):193-205 